\title{
Dynamic Imaging of the Fetal Heart Using Metric Optimized Gating
}

by

Christopher Roy

A thesis submitted in conformity with the requirements

for the degree of Master of Science

Department of Medical Biophysics

University of Toronto

(C) Copyright by Christopher William Roy 2012 


\title{
Dynamic Imaging of the Fetal Heart Using Metric Optimized Gating
}

\author{
Christopher Roy \\ Master of Science \\ Department of Medical Biophysics \\ University of Toronto
}

2012

\begin{abstract}
Advances in fetal cardiovascular MRI have been limited by the absence of a reliable cardiac gating signal. Recently, metric optimized gating (MOG) has been proposed as a solution to this limitation. In this thesis, I have developed and validated MOG for high resolution cine imaging of the fetal heart. ECG gated cine MR data of the adult heart were acquired from healthy volunteers. This enabled image reconstruction of the same data using both MOG and conventional ECG. Comparison of the two reconstruction methods was performed qualitatively, by comparing images reconstructed with each method, and quantitatively, based on the difference between MOG and ECG trigger times. Fetal images were also acquired, their quality evaluated by experienced radiologists, and the theoretical error in the MOG trigger times calculated. Excellent agreement between ECG and MOG reconstructions was observed. Using MOG, the world's first gated MR images of the human fetal heart were obtained. Small moving structures were visualized during radial contraction, thus capturing normal fetal cardiac wall motion and permitting assessment of cardiac function.
\end{abstract}




\section{Acknowledgements}

This work was supported through a studentship in part by the Ontario Opportunity Trust Fund - Hospital for Sick Children Foundation Student Scholarship Program and a Alexander Graham Bell Canada Graduate Scholarship from the Natural Sciences and Engineering Research Council of Canada.

To the members of my student committee, Mark Henkelman and Graham Wright, thank you for guiding, challenging, and supporting me throughout this project.

To Mike Seed, Bahiyah Al Nafisi, Lars Grosse-Wortmann, and Shi-Joon Yoo, thank you for your enthusiastic support. I feel extremely fortunate to be a part of the on-going collaboration between the clinical staff at Sick Kids and the Macgowan lab. Thank you Mike for your valuable insight, guidance, and frequent help acquiring and interpreting clinical data.

Thank you to my friends in the Macgowan lab: Peter Leimbigler, Chris Wernick and Joshua van Amerom. You have provided a fantastic work environment and valuable feedback at all stages of this project. Thank you Josh for all your help in and out of the lab.

I owe a tremendous amount of thanks to my supervisor Chris Macgowan. Without his guidance and support this project would not have been possible. Thank you for being an excellent teacher, friend, and for always giving me hours of your time when I ask for a couple seconds. I am extremely grateful to have had the chance to learn from you. 
To my friends Peter Beers and Nick Enright, though we no longer get to see each other on a regular basis, our discussions of life, science and music help keep me grounded and sane. To my friends and band mates Matt Horsman and Matt Mintzer, thank you for giving me a creative outlet outside of research.

To Victoria Munnoch, thank you for putting up with the endless hours I spend talking to my computer, and for always being there when I need you. This work, along with every aspect of my day to day life would not be possible without you.

Finally, to my incredible family, to my parents Paul and Maureen, my brothers Andrew and Corey and all of my relatives; thank you for your unwavering love and support. 


\section{Table of Contents}

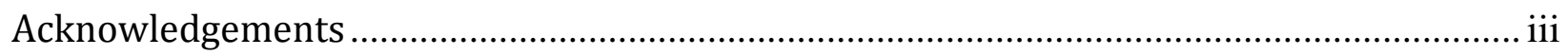

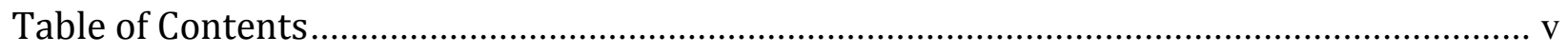

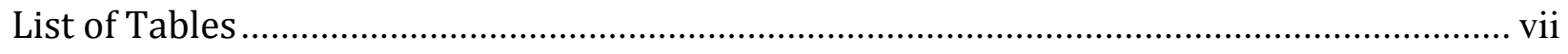

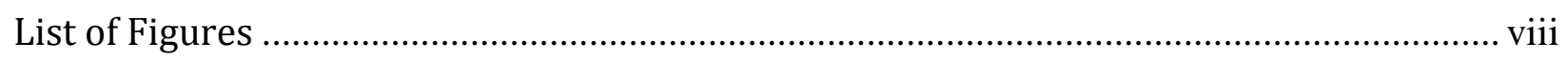

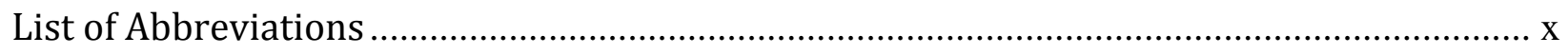

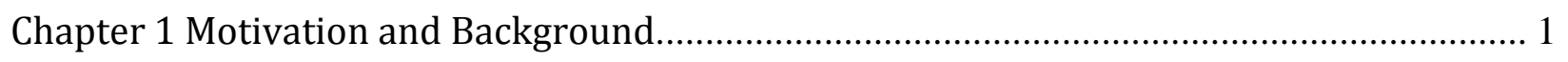

1.1 Imaging the Fetal Heart ........................................................................................... 1

1.1.1 Clinical Motivation - Fetal Distress ……………............................................. 2

1.1.2 Normal Circulation ......................................................................................... 2

1.1.3 Cardiac Function......................................................................................... 4

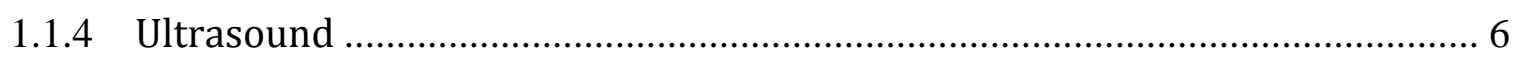

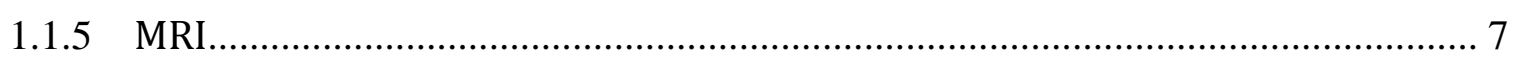

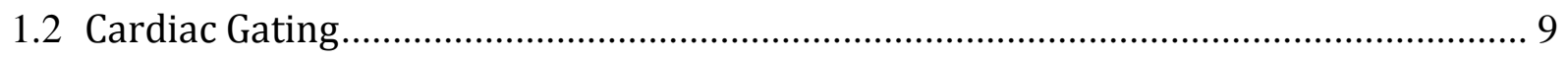

1.2.1 Conventional retrospective gating ............................................................... 9

1.2.2 Alternative Solutions.................................................................................. 10

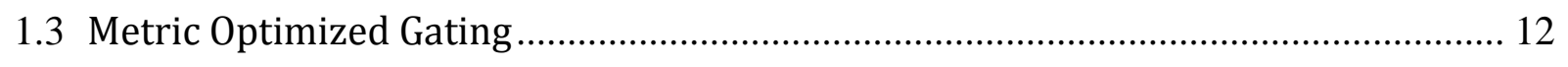

1.3.1 Metric-based Autocorrection ......................................................................... 12

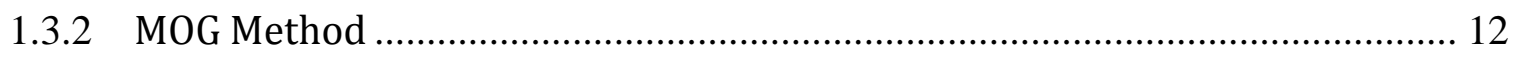

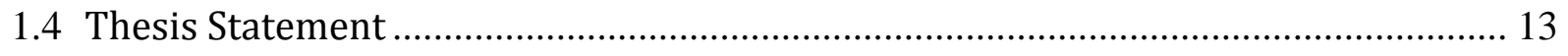

Chapter 2 Cine Imaging of the Heart Using Metric Optimized Gating ..................................... 15

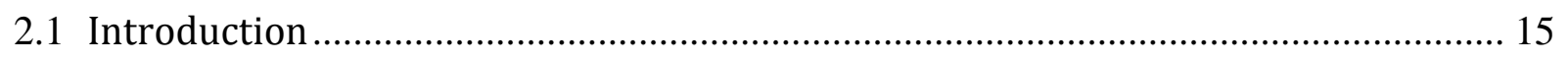

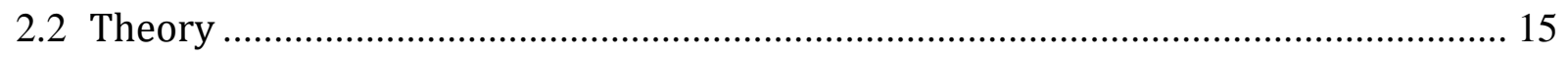

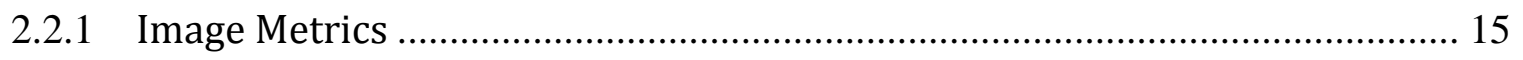


2.2.2 Hypothetical Waveform ................................................................................... 17

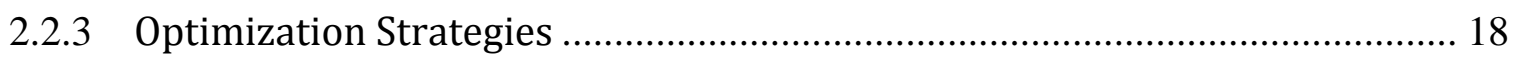

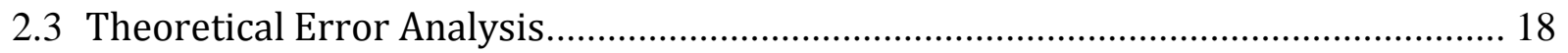

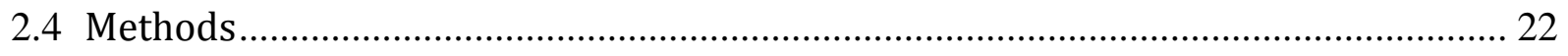

2.4.1 Adult Validation............................................................................................. 22

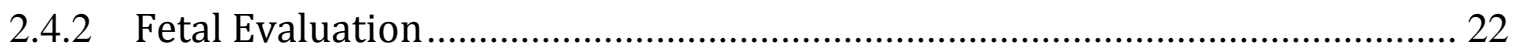

2.4.3 MOG Algorithm ...................................................................................... 24

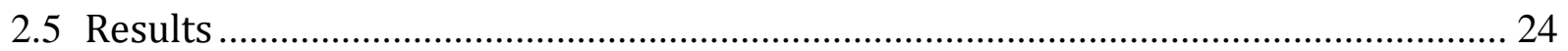

2.5.1 Adult Validation..................................................................................... 24

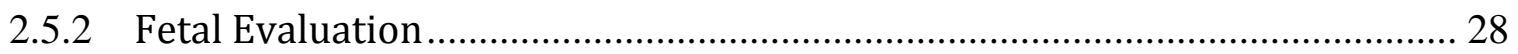

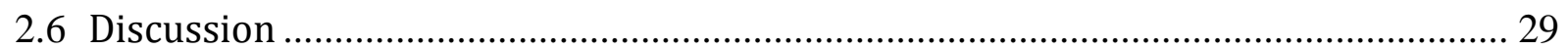

2.6.1 Validation ................................................................................................. 30

2.6.2 Fetal Application …………………………............................................ 30

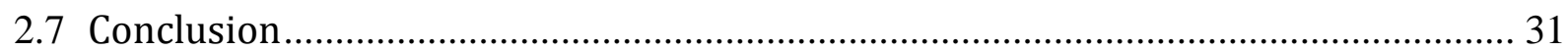

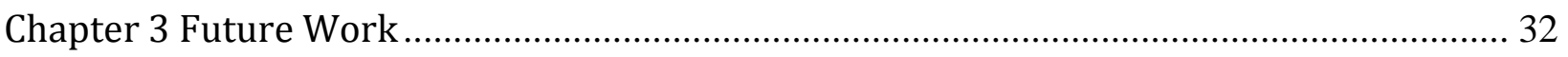

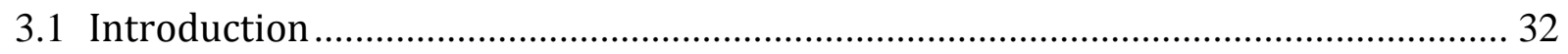

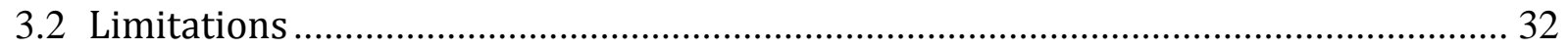

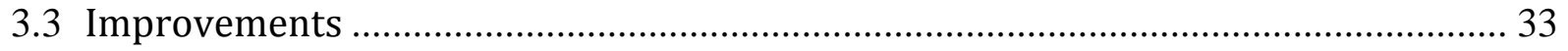

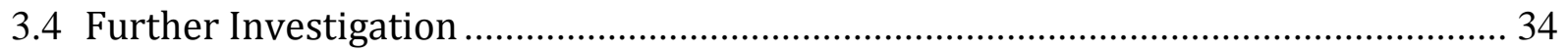

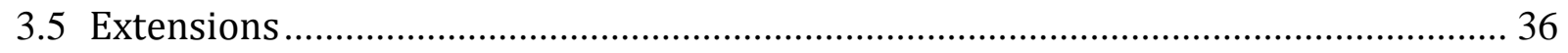

3.6 The Future of Fetal Imaging …………………….................................................... 36

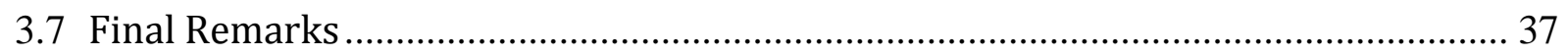

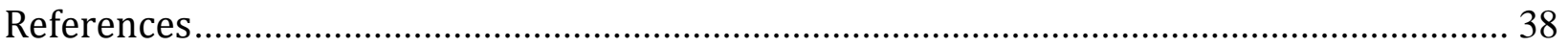




\section{List of Tables}

Table 1: Fetal cardiac function indices ........................................................................... 5

Table 2 Scan parameters for in vivo data acquisition....................................................... 23 


\section{List of Figures}

Figure 1: Diagram of the fetal circulation. Flow direction is indicated by the black arrows. Relative oxygen concentration is given by vessel color decreasing from red to blue (13). 4

Figure 2: Schematic of the MOG method. (a) K-space data are acquired, triggered by a synthetic waveform. (b) The data are retrospectively interpolated and combined according to a hypothetical waveform. Image reconstruction (2D inverse Fourier Transform) is performed and an image metric (entropy) quantifies artifact over a region of interest in the reconstructed images. The hypothetical waveform is adjusted and k-space interpolation, image reconstruction, and image metric calculation is repeated. (c) When the entropy is minimized, the final set of cine images is output.

Figure 3: The effect of misgating (a) Single frame from a retrospectively reconstructed set of adult images acquired with ECG gating and Cartesian sampling. (b) M-mode display of intensities along the vertical dash line in (a) showing the temporal evolution of the signal. (c, d) Data reconstructed with an incorrect estimate of the heart rate, showing a loss of both spatial and temporal resolution. 16

Figure 4 Entropy as a function of parameter offset. The theoretical error is given by the timing offset required to raise the entropy an amount $\sigma(\mathrm{E})$ above its minimum value. ...... 21

Figure 5: Comparison of MOG and ECG adult volunteer reconstructions. End-systolic (S) and diastolic (D) images are shown for each sampling strategy: (a, b) Cartesian without parallel imaging (c, d), Cartesian sampling with GRAPPA (e, f), and Radial sampling without parallel imaging. In all cases the temporal evolution of signal along the white dashed line is plotted..... 26

Figure 6: Theoretical error as a function of MOG parameter for data acquired using (a) Cartesian and (b) radial sampling. 27

Figure 7: Entropy as a function of timing offset. (a-c) Demonstration of the change in entropy that occurs when Cartesian k-space data are interpolated and reconstructed based on R-wave 
triggers that are shifted from their true ECG values. (d-f) shows the same process but for radial data.

Figure 8: MOG reconstructions of a healthy human fetal heart. (a) A short axis view at the mid ventricular level is shown at end-systole and end-diastole. (b) Magnified end diastolic image from (a). (c) Plot of signal intensity versus time for the dashed line in (b) 29

Figure 9: MOG reconstructions of human fetal subject with a congenital heart defect. (a) A short axis view at the mid ventricular level is shown at end-systole and end-diastole. (b) Magnified end diastolic image from (a). (c) Plot of signal intensity versus time for the dashed line in (b)........ 29

Figure 10: Preliminary results: ROI selection. (a) ROI example (dashed line), where the entropy is only evaluated over pixels that contain cardiac anatomy. (b) Demonstration of the change in entropy (evaluated over the ROI in a) that occurs when Cartesian k-space data are interpolated and reconstructed based on R-wave triggers that are shifted from their true ECG values. (c) ROI example (dashed line), where the entropy is evaluated over pixels extending 20\% beyond the cardiac anatomy in either direction. (d) Same as (b) but for the ROI shown in (c)..................... 35 


\section{List of Abbreviations}

2D/3D/4D Two/Three/Four dimensional

CMR Cadiovascular magnetic resonance imaging

ECG Electrocardiogram

FOV Field of view

GRAPPA Generalized Autocalibrating Partially Parallel Acquisition

MOG Metric Optimized Gating

MRI Magnetic resonance imaging

PC MRI Phase contrast magnetic resonance imaging

REB Research ethics board

ROI Region of interest

SENSE Sensitivity encoding

SNR Signal to noise ratio

SSFP Steady state free precesion

TR Repetition time

TE echo time

US Ultrasound 


\section{Chapter 1}

\section{Motivation and Background}

Since its inception over 30 years ago, the field of fetal magnetic resonance imaging (MRI) has grown considerably and now provides valuable tools for assessing the fetal anatomy including the brain, lungs, liver, and gastrointestinal tract (1-4). However, fetal cardiovascular MRI (CMR) remains relatively under-developed. CMR is an important tool for the non-invasive assessment of cardiac function, anatomy, and pathology in post-natal subjects. Advances in fetal CMR may therefore provide useful tools for the in utero assessment of normal fetal cardiovascular physiology and diseases that affect the fetal cardiovascular system. Potential applications of fetal CMR include congenital heart disease, placental insufficiency and other cardiovascular causes of fetal distress.

Unfortunately, the development of fetal CMR has been limited by practical challenges. This chapter provides an overview of the role of imaging in the assessment of both normal and diseased fetal cardiac physiology. The technical challenges of fetal CMR are introduced with a focus on cardiac gating. This chapter concludes with an overview of Metric Optimized Gating (MOG), a solution to fetal cardiac gating. Previous work in MOG applied to velocitysensitive MRI measurements of fetal blood flow is introduced, leading to the focus of this work: the development and validation of MOG for cine MR imaging to characterize chamber function of the human fetal heart.

\subsection{Imaging the Fetal Heart}

During pregnancy, cardiac imaging is used to monitor the developing fetus. In high risk pregnancies or those suspected of having fetal distress, imaging provides vital information for patient management, enabling physicians to make the best possible choices regarding delivery, antenatal, and perinatal treatment. To appreciate the role imaging plays in assessing both the normal and compromised fetal circulation, it is important to understand the key differences between fetal and post-natal cardiac physiology. The following provides an overview of fetal distress and the fetal circulatory system. Given the application of this 
work (cine MR imaging of the fetal heart), an emphasis is put on the chambers of the heart and how cardiac function is assessed.

\subsubsection{Clinical Motivation - Fetal Distress}

This work is motivated by the need for quantitative imaging methods in the assessment of fetal distress. Fetal distress is defined as progressive fetal asphyxia where the supply of oxygen to the body is severely decreased. If not corrected or circumvented by early delivery, fetal distress results in failure of the cardiovascular system to redistribute blood flow adequately and preserve oxygenation of vital organs. This may cause permanent damage to the central nervous system and other organs, or death (5). In response to signs of fetal distress, medical inductions of labor and caesarean sections are becoming more common. Preterm births in North America have increased from approximately $9 \%$ of all births in 1980 to $12.3 \%$ in $2010(6,7)$. Conventional clinical testing has improved the detection of fetal distress but remains non-specific, and many infants with suspected fetal distress are delivered early unnecessarily (8). Preterm birth is the leading cause of newborn death, and babies who survive often have lifelong complications, including breathing problems, cerebral palsy, and cognitive disabilities (9). Babies born just a few weeks early have higher rates of hospitalization and illness than full-term babies (10). In cases of suspected fetal distress, physicians are faced with a difficult decision of whether to deliver the baby early or to allow the pregnancy to progress naturally. They must weigh the risk of brain injury (caused by a lack of oxygen to the fetal brain) against the significant problems associated with premature birth. To properly deliver or treat a fetus that is suspected to be in distress, accurate methods for assessing cardiac function are needed. To track pathological changes as they relate to fetal distress we must first understand the normal fetal circulation. The following section describes the normal circulation and how it differs from post-natal life.

\subsubsection{Normal Circulation}

In the normal fetal circulation illustrated in Figure 1, well-oxygenated blood returning from the placenta through the umbilical vein is split between the liver and the ductus venosus. Most of the umbilical vein blood is thought to bypass the liver and is directed into the 
thoracic portion of the inferior vena cava via the ductus venosus. Umbilical vein blood that does enter the left liver combines with small amounts from the hepatic artery. The result is relatively well oxygenated blood in the left liver. Conversely, umbilical vein blood entering the right liver combines with poorly oxygenated portal venous blood, and results in relatively less oxygenated blood in the right liver $(3,11)$. Blood from the left and right hepatic veins rejoins blood from the ductus venosus and enters the inferior vena cava. The inferior vena cava then contains a mixture of well oxygenated blood, from the left liver and ductus venosus, and less oxygenated blood from the right liver and abdominal portion of the inferior vena cava.

Blood from the inferior vena cava enters the right atrium where it is joined by blood from the superior vena cava. Here, the Eustachian valve and lower portion of the atrial septum move together to direct blood through the foramen ovale or through the tricuspid valve. Blood passing across the foramen ovale and into the left atrium has an oxygen saturation of approximately $65 \%$; while slightly less oxygenated blood $(\sim 55 \%)$ enters the right ventricle through the tricuspid valve. Blood in the left atrium combines with the slightly less oxygenated blood from the left pulmonary vein, enters the left ventricle through the mitral valve, and is ejected into the ascending aorta to feed the coronary, cerebral and upper body circulation. Although some blood from the right ventricle enters the pulmonary circulation, most is directed via the ductus arteriosus into the descending ascending aorta, supplying the placenta, via the umbilical arteries, and the lower body (11).

There are two key differences between the post natal and fetal circulation systems. First, the site of gas exchange in post-natal life is the lungs as opposed to the placenta in utero. Second, the post-natal circulation is comprised of two circulations, pulmonary and systemic, operating in series. The pulmonary circulation exchanges blood between the heart and lungs while the systemic circulation distributes blood between the heart and the rest of the body. As a consequence, there is no mixing of oxygen rich pulmonary venous blood with less rich systemic venous blood. In contrast, the two fetal circulations are described as left and right circulations that act in parallel and contain several sites where blood with different oxygen concentrations mix (12). 


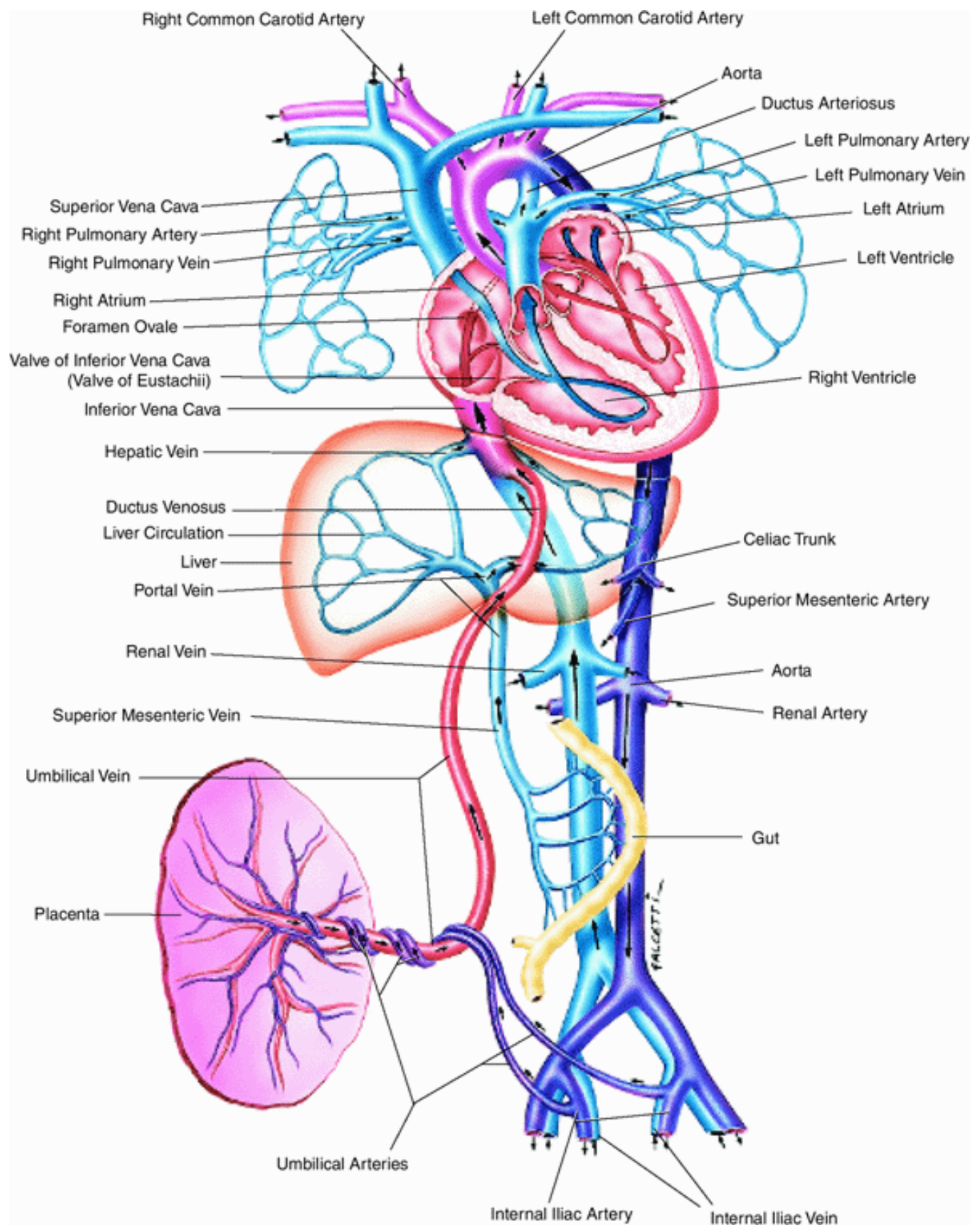

Figure 1: Diagram of the fetal circulation. Flow direction is indicated by the black arrows.

Relative oxygen concentration is given by vessel color decreasing from red to blue (13).

\subsubsection{Cardiac Function}

The previous section described the differences in both the structure and function of the post-natal and fetal circulations. Despite these differences, several imaging-based methods 
for assessing the post-natal heart have been successfully adapted for evaluation of the fetal heart. These include flow based measurements and methods for identifying the cardiac anatomy. The following briefly describes the components of the normal heart and how their function is measured with modern imaging techniques.

In the normal fetal circulation described by Figure 1, the fetal heart is comprised of a left and right pump. Each pump consists of an atrium that receives venous blood and a ventricle that eject blood into an arterial system. If the function of either of these pumps is impaired, the fetal circulation may be compromised leading to fetal distress as described above. Therefore, several different indices have been borrowed from general cardiology for quantitative evaluation of cardiac function. These include stroke volume, cardiac output, ejection fraction, and shortening fraction (specific formulae given by Table 1). From Table 1 we see that these methods can be summarized as ratios of blood volume that enter and are ejected by the two cardiac pumps. Therefore, an important aspect of evaluating cardiac function involves the measurement of blood flow in the cardiac chambers during systole (contraction) and diastole (relaxation). This may be performed by direct volumetric measurement or a surrogate measurement (i.e. blood flowing in a major vessel) using the modern medical imaging techniques described in the following sections (14).

Table 1: Fetal cardiac function indices

\begin{tabular}{lc}
\hline Index & Formula \\
\hline Stroke volume (SV) & EDV-ESV \\
Cardiac output & $\mathrm{SV} \times$ heart rate \\
Ejection fraction & $\mathrm{SV} / \mathrm{EDV}$ \\
Shortening fraction & (EDVD-ESVD)/EDVD \\
\hline
\end{tabular}

EDV, end-diastolic volume; ESV, end-systolic volume; EDVD, end-diastolic ventricular diameter; ESVD, end-systolic ventricular diameter 


\subsubsection{Ultrasound}

Ultrasound (US) is the standard imaging modality for assessment of the fetal heart with normal values in the fetus first presented in 1982 (15). It is non-invasive, relatively cheap, accessible, capable of real-time imaging at high spatial resolution, and does not involve ionizing radiation. Ultrasound evaluation of cardiac function includes M-mode echocardiography (15), Doppler flow mapping (16), and three and four-dimensional (3D/4D) ultrasound (17). M-mode echocardiography involves the temporal analysis of two-dimensional (2D) cardiac structures as they interact with an ultrasound beam. While the 2D nature of M-mode scanning prevents volumetric measurements, the shortening fraction, a well established surrogate of cardiac function, can be determined (18). Doppler flow mapping can also be used to provide surrogate measurements of volumetric flow. The stroke volume of the left heart is measured with Doppler using the diameter of the aorta multiplied by the flow across the valve annulus. Similarly, the right stroke volume is calculated from the diameter of the pulmonary artery and the related flow (19). Recent studies in 3D/4D ultrasound have enabled direct quantification of ventricular volumes and thus provide measurements of stroke volume, cardiac output and ejection fraction (20).

These methods are however not without limitations. 2D US measurements of the fetal cardiac chambers are limited by their geometric assumptions needed to calculate ventricular volumes. In particular, the irregular shape of the right ventricle makes accurate volumetry, and hence evaluation of cardiac function, a challenge (21). Advances in 3D/4D US have shown improved reproducibility when compared to 2D US but further evaluation is needed to demonstrate its accuracy. Finally, US measurements require an acoustic window to the target that may be limited by low amniotic fluid levels, adverse fetal position, or maternal obesity (22). Despite these limitations, ultrasound provides excellent techniques for assessing cardiac function. Still, several studies have suggested that in utero MRI may provide a useful complement to ultrasound (14,23-25). 


\subsubsection{MRI}

MRI scanning of pregnant women was first reported by Smith et al. in 1983 (26). In their work, pregnant women were imaged to diagnose maternal and placental abnormalities. In utero fetal MRI studies first attempted to measure volumes from echo planar images but often suffered in quality due to fetal movement during the scan (27-29). The advent of rapid acquisition sequences has led to a number of successful volumetry and functional studies in the fetal lungs, liver, gastrointestinal tract and brain (1-4). In particular, the fetal central nervous system has been the focus of many studies, from which fetal MRI has been shown to provide valuable additional diagnostic information to that of ultrasound alone (4). In recent years, the field of fetal MRI has grown considerably, and provided valuable tools for assessing the fetal anatomy. One area that has lacked development however is fetal cardiovascular MRI (CMR).

\section{$\underline{\text { CMR }}$}

CMR potentially offers a wide range of tools for assessing the fetal heart. It is non-invasive, provides excellent soft tissue contrast and has the ability to perform multi-planar imaging in any orientation. Phase contrast (PC) MRI is the gold standard for non-invasive measurement of blood flow in post-natal subjects and similarly, steady state free precession (SSFP) cine MRI is the post-natal reference standard for ventricular volumetry $(30,31)$. The application of these methods to fetal subjects in utero would provide quantitative evaluation of cardiac function that is analogous to ultrasound-based measurements, thus providing complementary information to echocardiography in situations where ultrasonic evaluation is limited or not possible. Furthermore, MRI techniques exist for estimating the oxygen saturation in blood which when combined with PCMRI and SSFP cine imaging may provide a novel method for accurately evaluating the fetal circulation $(32,33)$.

Unfortunately, in utero MRI assessment of the fetal heart has been limited by practical challenges. During late gestation, the diameter of a normal left ventricle is only $15 \mathrm{~mm}$ and major vessels are even smaller with diameters less than $8 \mathrm{~mm}$ (34). These small 
dimensions coupled with high heart rates (between 110-180 bpm) necessitate high spatial and temporal resolutions to capture cardiac dynamics (35). Resolution in fetal scans has been constrained by two main factors. First, a large field of view (FOV) is needed to avoid image wrap from the maternal abdomen and second, the possibility of fetal motion during the examination puts considerable constraint on scan time.

For simplicity, the following assumes Cartesian sampling when describing these trade-offs; however, these concept extend to alternative sampling strategies (radial, spiral, etc). In conventional MRI, the spatial resolution of an image is equal to the FOV divided by the number of rows that make up the Fourier data or k-space matrix. In dynamic imaging, each $\mathrm{k}$-space matrix is acquired multiple times and the temporal resolution of the resulting CINE images is equal to the acquisition time of a single row of k-space (TR). Scan time depends on the total number of acquired k-space rows and thus for a given FOV, a trade-off exists between spatial resolution, temporal resolution and scan time.

Several strategies exist for accelerating MRI acquisitions and represent a large field of active research. Using these strategies, one can shorten scan time or, improve spatial/temporal resolution. While a full review of acceleration strategies is beyond the scope of this thesis, two methods, segmented k-space and parallel imaging, were used to shorten the MRI acquisitions presented in Chapter 2 and are described below.

In a segmented MRI acquisition, rather than sampling each row in repeated fashion, a group of rows or "segment" of k-space is repeatedly sampled. The temporal resolution is then equal to TR multiplied by the number of rows (views) per segment. Increasing the number of views per segment shortens scan time but at the cost of temporal resolution (36).

Parallel imaging describes a number of techniques that use spatial information from phased-array coils to reduce acquisition times. Here, the number of k-space rows acquired is reduced by a factor $\mathrm{R}$ but spatial resolution is maintained. As a result the field of view is decreased and aliasing occurs. Depending on the method, extra lines of k-space are 
acquired before or during the image acquisition to calibrate coil sensitivities and their spatial dependence is later used to reconstruct the missing k-space rows and remove aliasing. Existing parallel imaging schemes differ in the way they calibrate coil sensitivities and reconstruct the missing rows (37). Several parallel imaging schemes have been proposed but the most widely available are generalized auto-calibrating partially parallel acquisition (GRAPPA) and sensitivity encoding (SENSE). In GRAPPA, calibration rows are acquired during the scan (38). These rows are used to generate the missing rows before image reconstruction (Fourier transform). SENSE uses a separate acquisition to obtain coil sensitivity profiles. The under-sampled k-space is Fourier transformed, and aliasing is removed from the images using the coil sensitivity profiles (39).

Various combinations of the above mentioned methods as well as emerging techniques such as compressed sensing and reduced field of view imaging have been described in the literature $(40,41)$. Combining acceleration strategies so that both temporal and spatial undersampling is performed allows for dramatic reductions in scan time (42-44). Conventional CMR scans, even with aggressive acceleration, take place over several heart beats. To improve both temporal and spatial resolution, it is then necessary to acquire and retrospectively combine MRI data from multiple cardiac cycles (45). In adults, this is accomplished by synchronizing the data acquisition to an electrocardiogram (ECG) waveform through a process known as cardiac gating. The following section describes cardiac gating, including conventional retrospective gating for post-natal subjects and alternative gating techniques that have been proposed for fetal imaging.

\subsection{Cardiac Gating}

\subsubsection{Conventional retrospective gating}

In retrospectively gated scans of post-natal subjects, an ECG waveform "triggers" the continuous acquisition of data. Here, triggering refers to collecting data upon the detection of the ECG waveform. The data are then retrospectively sorted. For each segment of kspace, the time point in the cardiac cycle (cardiac phase) is determined according to the acquisition time with respect to the last ECG trigger. Data are then interpolated to a 
uniformly distributed set of cardiac phases and Fourier transformed to create a set of images spanning a representative heart beat (46). Cardiac gating is important in that it allows one to "freeze" the cardiac anatomy at a specific cardiac phase. If a stack of cine images are acquired, 3D volumetry can be performed at end-systole and end-diastole allowing for cardiac function evaluation as described above. Given the importance of cardiac gating, the development of fetal CMR has been drastically limited by the difficulty in measuring a fetal electrocardiogram (ECG) signal for cardiac gating (47). Unfortunately, the fetal ECG signal is typically an order of magnitude weaker than that of the mother, and can be difficult to detect in the presence of electrical noise from the MRI (48).

\subsubsection{Alternative Solutions}

Current alternative strategies for fetal CMR include ultrasound based cardiac monitoring, self-gating, real time imaging, and synthetic gating. These strategies are described below followed by the technique presented in this work: Metric Optimized Gating (MOG).

\section{Fetal Cardiotocography}

Non-imaging ultrasound (fetal cardiotocography) is an established technique for monitoring the fetal heart rate. MR-compatible units show promise for fetal cardiac gating, although they can be limited by interference between the unit and MR system (49). Furthermore, the likelihood of fetal movement during the scan may challenge the reliability of the gating signal. Fetal cardiotocography has been used to image the heart of fetal sheep under anaesthesia but no direct human application has been published to date (50).

\section{Self-gating}

Another approach to fetal cardiac gating is MRI "self gating", where a periodic gating signal is extracted from the MR data itself and is used to sort the data retrospectively (51). Modern implementations exist for both radial and Cartesian trajectories, and use a variety of signal detection algorithms (52-54). Self gating is attractive as it requires no additional hardware, although the reliability of the gating signal depends on slice prescription and temporal resolution, and therefore may not accommodate all k-space sampling trajectories. 
Previous publications have used self-gating for cardiac imaging in the fetal mouse, chick, and lamb (55-57). These techniques may be applied to human fetal CMR, but as with ultrasound gating, no human application has been published to date.

\section{$\underline{\text { Real-time MRI }}$}

In real-time CMR, an entire frame of k-space is acquired in a single shot. The temporal resolution of the resulting set of images is then equal to the time required to acquire a single frame. A number of frames are acquired to cover the full cardiac cycle. Seiberlich et al. 2011 have recently demonstrated a real time method that combines aggressive parallel imaging with a non-Cartesian k-space sampling trajectory (58). While they were able to achieve excellent temporal resolution $(\sim 20-35 \mathrm{~ms})$ their FOV $\left(30 \times 30 \mathrm{~cm}^{2}\right)$ and spatial resolution $(2.4 \mathrm{~mm})$ is inadequate for fetal imaging.

\section{Synthetic Gating}

In the absence of an ECG or alternative gating signal, cine images can be acquired using a synthetic waveform to periodically trigger the data acquisition. Using this approach, "pseudogating" can be performed by estimating the mean heart rate of the subject (59). If the heart rate is approximately constant and the estimate is accurate, then every segment of k-space is sampled at each phase of the cardiac cycle. These data can then be reconstructed in the same manner as retrospectively gated data with the heart rate estimate replacing the ECG waveform. However, if the true heart rate varies throughout the scan, the acquired k-space segments will be incorrectly combined based on an inaccurate calculation of the cardiac phase for each segment and the resulting images will contain artifact. In this thesis, temporal misalignment of k-space data and the resulting artifact is referred to as "misgating". An alternative synthetic gating strategy is to purposefully oversample each cardiac cycle, by setting the period of the gating waveform to be longer than the fetal cardiac cycle $(>500 \mathrm{~ms})(54)$. This guarantees that every row of k-space is sampled at each phase of the cardiac cycle but the cardiac phase associated with each segment remains unknown and, thus, the resulting reconstructions will be misgated. 


\subsection{Metric Optimized Gating}

The approach to fetal gating used in this thesis is called Metric Optimized Gating (MOG) (60). MOG was developed to retrospectively combine synthetically-gated, oversampled data. Oversampled data acquisitions were chosen to ensure that even though the cardiac phase of each segment is unknown, every row of k-space is sampled at each phase of the cardiac cycle. To combine oversampled data but avoid image degradation due to misgating, an iterative method was created based on previous work in metric-based autocorrection.

\subsubsection{Metric-based Autocorrection}

Previous investigators have employed metric-based autocorrection to remove artifact from images in several fields including photography, astronomy, and medical imaging. The most relevant applications for this thesis stem from work performed by Atkinson et al. and further refined by McGee et al. (61,62). In their work, k-space data were iteratively adjusted to remove motion artifact from images of the brain, shoulder and angiography $(61,63,64)$. In all cases a cost function or metric is used to determine the optimum image quality. A number of image metrics are described by these authors and their application in this work is further discussed in Chapter 2.

\subsubsection{MOG Method}

As with the metric-based autocorrection methods described above, MOG employs an iterative process that corrects images corrupted by cardiac motion. This iterative process is depicted in Figure 2. Upon collecting synthetically-gated, oversampled data, a hypothetical gating waveform is created that spans the period of image acquisition. Images are reconstructed using this hypothetical sorting information, following the same steps described above for cardiac gating. Artifact from misgating is quantified using a metric for image quality, calculated over a region of interest (ROI) in the image, and the relative timings of the hypothetical triggers are adjusted. The process is repeated iteratively until the image metric is optimized, and the optimum set of cine images are output. 


\subsection{Thesis Statement}

MOG was first developed in our lab to resolve fetal blood flow measurements using PCMRI. The purpose of this thesis is to develop and validate Metric Optimized Gating for cine imaging of the fetal heart. In the next Chapter I propose a technical improvement to the MOG technique and develop a novel error analysis method for comparing MOG to the gold standard, ECG gating. The application of MOG to anatomical imaging enables evaluation of fetal cardiac function. When combined with previous work in MOG applied to fetal blood flow measurements, this work provides an important step towards a comprehensive in utero MRI assessment of fetal hemodynamics. This will hopefully lead to improved patient management in pregnancies with suspected fetal distress. 
Acquire oversampled k-space data triggered by a synthetic waveform

a Data acquisition
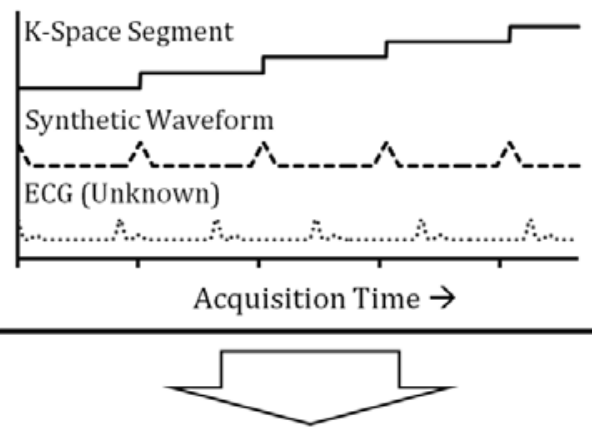

Create hypothetical Waveform

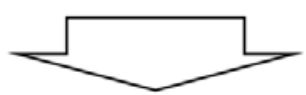

b Intermediate data analysis
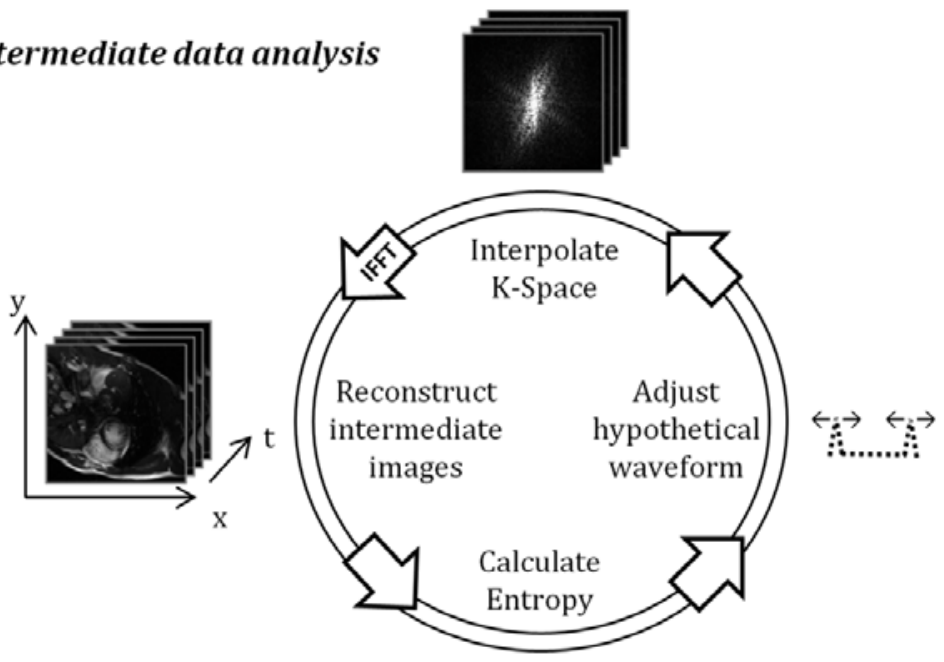

$$
\mathrm{E}=\Sigma_{\mathrm{t}} \frac{\mathrm{B}_{\mathrm{t}}}{\sum_{\mathrm{t}} \mathrm{B}_{\mathrm{t}}} \Sigma_{\mathrm{x}} \Sigma_{\mathrm{y}} \frac{\mathrm{S}_{\mathrm{x}, \mathrm{y}, \mathrm{t}}}{\mathrm{B}_{\mathrm{t}}} \log \left(\frac{\mathrm{S}_{\mathrm{x}, \mathrm{y}, \mathrm{t}}}{\mathrm{B}_{\mathrm{t}}}\right)
$$

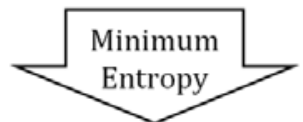

c Optimized output
Figure 2: Schematic of the MOG method. (a) K-space data are acquired, triggered by a synthetic waveform. (b) The data are retrospectively interpolated and combined according to a hypothetical waveform. Image reconstruction (2D inverse Fourier Transform) is performed and an image metric (entropy) quantifies artifact over a region of interest in the reconstructed images. The hypothetical waveform is adjusted and $\mathrm{k}$ space interpolation, image reconstruction, and image metric calculation is repeated. (c) When the entropy is minimized, the final set of cine images is output. 


\section{Chapter 2 \\ Cine Imaging of the Heart Using Metric Optimized Gating 1}

\subsection{Introduction}

In the previous chapter, the role of imaging in the context of normal and compromised fetal circulation was presented. Image-based evaluation of cardiac function was introduced and the technical challenges (image resolution, cardiac gating) that limit in utero CMR were discussed. In this Chapter, I present the theory and implementation of Metric Optimized Gating (MOG) for cine MR images of the fetal heart. This method exploits the fact that nongated cardiac MR images contain characteristic artifacts that can be quantified using an image metric. I present healthy adult volunteer data for validation, develop a novel method for error analysis of the MOG technique, and finally obtain the first gated high-resolution cine images of the fetal human heart in both a healthy subject and one with suspected fetal distress.

\subsection{Theory}

The application of the MOG technique to cine imaging of the fetal heart presents three challenges. First, an image metric is required that is sensitive to the effects of misgated cine images of the fetal heart. Second, a method for constructing a hypothetical waveform, which provides retrospective sorting information in the MOG algorithm, must be developed. Finally, a metric minimization algorithm is required to optimize the hypothetical waveform in a practical processing time.

\subsubsection{Image Metrics}

While a number of different image metrics have been proposed in the literature, the image entropy and the entropy of the gradient of the image are prevalently identified as appropriate metrics for quantifying motion artifact (62). McGee et al. describe the entropy

\footnotetext{
${ }^{1}$ This chapter is derived from a manuscript that has been submitted to Magnetic Resonance in Medicine
} 
of the gradient as an appropriate metric for images wherein high resolution structures are distorted within the image. Conversely, the image entropy is appropriate for images with ghosting outside the moving object (65). Atkinson et al. further state that entropy minimization removes blurring from structures that would otherwise be dark (61). Given that the fetal myocardium and surrounding anatomy is naturally dark relative to the bright blood pool in SSFP images, the image entropy is potentially a good metric for identifying artifacts in misgated cardiac SSFP images. In misgated cardiac images the sources of artifact are blurring and ghosting. Figure 3 illustrates the effective decrease in temporal resolution that appears when comparing gated and misgated image reconstructions. There is also a decrease in contrast between the bright blood pool and otherwise dark surrounding tissue. As previously mentioned, the image entropy is sensitive to ghosting and blurring in areas of an image that should otherwise be dark, thus confirming that entropy is a suitable metric for this application (61). In this work the entropy is calculated using the following expression:

$$
E=\sum_{t} \frac{B_{t}}{\sum_{t} B_{t}} \sum_{x} \sum_{y} \frac{S_{x, y, t}}{B_{t}} \log \left(\frac{S_{x, y, t}}{B_{t}}\right),
$$

where $S$ is the pixel intensity with $\mathrm{x}$, $\mathrm{y}$ indexing over space, $\mathrm{t}$ indexing over time, and $\mathrm{B}$ is a normalization factor adapted from Atkinson et al.(61):

$$
B_{t}=\left[\sum_{x} \sum_{y} S_{x, y, t}^{2}\right]^{1 / 2}
$$
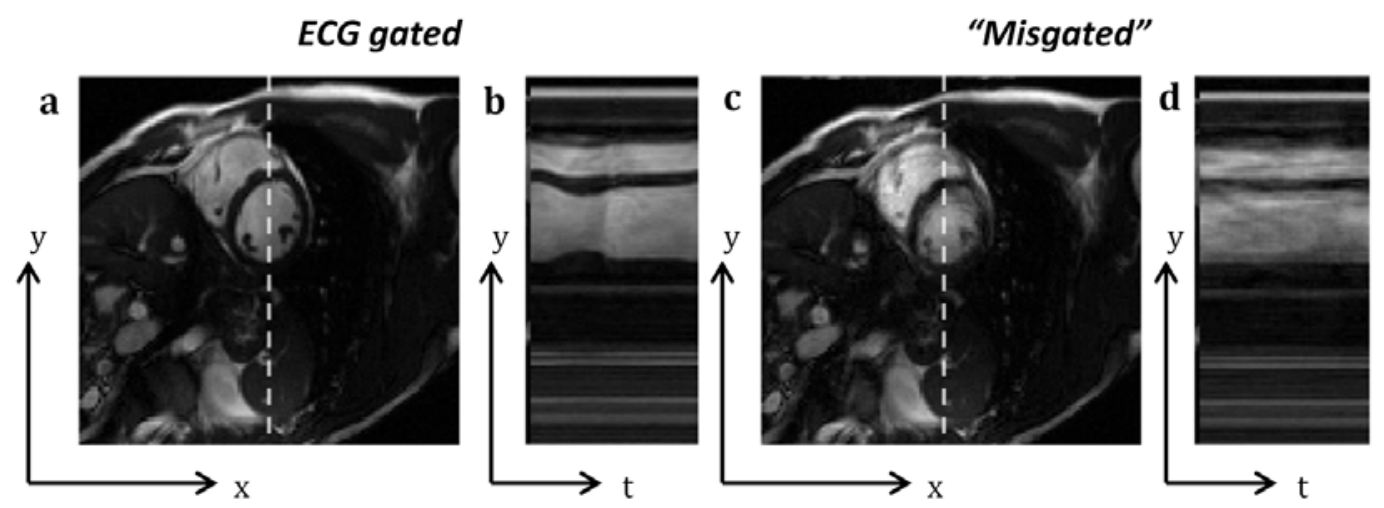

Figure 3: The effect of misgating (a) Single frame from a retrospectively reconstructed set of adult images acquired with ECG gating and Cartesian sampling. (b) M-mode display of intensities along the vertical dash line in (a) showing the temporal evolution of the signal. (c, d) Data reconstructed with an incorrect estimate of the heart rate, showing a loss of both spatial and temporal resolution. 


\subsubsection{Hypothetical Waveform}

Recall that in the MOG method, a hypothetical waveform replaces conventional ECG gating. The corresponding hypothetical triggering information is then used to retrospectively reconstruct a set of images. In order to optimize this triggering information, a parameterized model of the subject's heart rate (i.e. period between hypothetical triggers) is required. The simplest possible model would consist of a single parameter representing a constant heart rate that spans the length of the image acquisition. Conversely a model consisting of many parameters would represent each hypothetical trigger individually.

For a given model there is an inherent trade-off between flexibility (ability to account for beat to beat heart rate variation) and post-processing time (time required to optimize each parameter). The initial development of MOG in our lab used a piece-wise model that assumed the subject's heart rate (i.e. the period between triggers) was constant for a portion of the data acquisition. In this model, each parameter represented the subject's heart rate over a portion of the image acquisition (i.e. a two parameter model would assume a constant heart rate for each half of the data acquisition). Jansz et al. demonstrated that a two parameter piece-wise constant model was sufficient for MOG applied to PCMRI measurements of blood flow, providing some model flexibility while maintaining a practical post-processing time (60). However, their results showed a bias towards agreement between their model and the true heart rate for triggers that took place near the midpoint of the scan. Consequently, data that were collected at the beginning and end of the scan may have been misgated in the presence of high heart rate variability. Still, fetal blood flow measurements provided by Jansz et al. showed good reproducibility, suggesting that the heart rate model need only be flexible near the midpoint of the scan, which for Cartesian sampling, represents high-powered low resolution k-space data.

For cine imaging of the heart, dynamic high resolution structures exist that may necessitate gating that is accurate and unbiased for all portions of k-space. Therefore, despite the success of the two-parameter model for MOG applied to PCMRI, the hypothetical gating waveform used in this work is a multi-parameter model with each parameter representing 
the timing of one trigger. While this model provides excellent flexibility in the presence of fetal heart rate variation, post-processing times are inherently longer. To address this, I have explored alternative optimization strategies that minimize the image entropy in fewer iterations.

\subsubsection{Optimization Strategies}

The simplest method for optimizing multiple parameters is through a brute force search that calculates the cost function (entropy) for every combination of possible parameter values. This method is computationally intensive but has the advantage of being exhaustive, thereby increasing the confidence in the minimum. Conversely, more efficient optimization algorithms evaluate fewer parameter combinations but require the cost function to vary smoothly and to be well behaved to avoid solutions at local minima (66). Efficient optimization routines represent a wide body of research. The Nelder-Mead algorithm in particular is widely used in the fields of chemistry and medicine for multiparameter unconstrained optimization (67). This method is attractive in that it does not require any derivative calculations but instead performs a simplex based search where the optimum value is reached when the vertices of the simplex (cost-function values) are minimized to a pre-determined termination value. Unlike gradient-based methods the Nelder-Mead simplex algorithm does not necessitate a smooth continuous function however, if the global minimum is not well behaved convergence may occur at local minima. I demonstrate later that the conditions for efficient optimization are experimentally satisfied for the MOG method.

\subsection{Theoretical Error Analysis}

The previous section has outlined three developments for the application of MOG to cine imaging of the fetal heart. Later in this work, validation of these developments is performed by comparing MOG and ECG reconstructions of healthy adult volunteer data. This is performed through a comparison of image quality and quantitatively by comparing MOG and ECG trigger times. Comparison of the MOG and ECG trigger times yields an experimental error in the MOG parameters. However a theoretical error analysis is also 
possible. For a given parameter, the uncertainty in the image entropy is determined by propagating the image noise through Eq. (1). The uncertainty in the image entropy is then transformed to provide an estimate of the theoretical error in each MOG parameter, based on the curvature of the entropy landscape about the optimum. To simplify several steps in the error propagation calculation below, Eq. (1) is written as follows:

$$
\mathrm{E}=\sum_{\mathrm{t}} \sum_{\mathrm{i}} \frac{\mathrm{S}_{\mathrm{i}, \mathrm{t}}}{\mathrm{C}} \cdot \ln \left(\frac{\mathrm{S}_{\mathrm{i}, \mathrm{t}}}{\mathrm{B}_{\mathrm{t}}}\right) \text {. }
$$

Here, the spatial indices $x$ and $y$ have been combined into one spatial coordinate $i . S_{i, t}$ is the intensity of the magnitude image at spatial location $\mathrm{i}$ and time $\mathrm{t}$ and a third variable $\mathrm{C}$ is introduced:

$$
C=\sum_{t} B_{t}
$$

Now, we define a function of three variables $e_{i, t}=e\left(S_{i, t}, B_{t}, C\right)$ :

$$
e\left(S_{i, t}, B_{t}, C\right)=\frac{S_{i, t}}{C} \cdot \ln \left(\frac{S_{i, t}}{B_{t}}\right)
$$

Eq. (3) is then written as:

$$
E=\sum_{\mathrm{t}} \sum_{\mathrm{i}} \mathrm{e}_{\mathrm{i}, \mathrm{t}}
$$

Hereafter $\sigma$ is used to denote the uncertainty of each expression. The uncertainty in E, $\sigma(E)$, then becomes:

$$
\sigma(E)=\sqrt{\sum_{\mathrm{t}} \sum_{\mathrm{i}}\left(\sigma\left(\mathrm{e}_{\mathrm{i}, \mathrm{t}}\right)\right)^{2}}
$$

The uncertainty in $e_{i, t}$ is given by:

$$
\sigma\left(\mathrm{e}_{\mathrm{i}, \mathrm{t}}\right)=\sqrt{\left(\frac{\partial \mathrm{e}_{\mathrm{i}, \mathrm{t}}}{\partial \mathrm{S}_{\mathrm{i}, \mathrm{t}}} \cdot \sigma\left(\mathrm{S}_{\mathrm{i}, \mathrm{t}}\right)\right)^{2}+\left(\frac{\partial \mathrm{e}_{\mathrm{i}, \mathrm{t}}}{\partial \mathrm{B}_{\mathrm{t}}} \cdot \sigma\left(\mathrm{B}_{\mathrm{t}}\right)\right)^{2}+\left(\frac{\partial \mathrm{e}_{\mathrm{i}, \mathrm{t}}}{\partial \mathrm{C}} \cdot \sigma(\mathrm{C})\right)^{2}} .
$$

The error in $S_{i, t}$ is the image noise in space and time. The uncertainties in $B_{t}$ and $C$ are given by the following:

$$
\sigma\left(B_{t}\right)=\sqrt{\frac{\sum_{i}\left(S_{i, t} \cdot \sigma\left(S_{i, t}\right)\right)^{2}}{\sum_{i} S_{i, t}{ }^{2}}} .
$$




$$
\sigma(\mathrm{C})=\sqrt{\sum_{\mathrm{t}}\left(\frac{\sum_{\mathrm{i}}\left(\mathrm{S}_{\mathrm{i}, \mathrm{t}} \cdot \sigma\left(\mathrm{S}_{\mathrm{i}, \mathrm{t}}\right)\right)^{2}}{\sum_{\mathrm{i}} \mathrm{S}_{\mathrm{i}, \mathrm{t}}{ }^{2}}\right)} .
$$

The partial derivatives of $e_{i, t}$ are:

$$
\begin{gathered}
\frac{\partial \mathrm{e}_{\mathrm{i}, \mathrm{t}}}{\partial \mathrm{S}_{\mathrm{i}, \mathrm{t}}}=\frac{1}{\mathrm{C}} \cdot\left(\ln \left(\frac{\mathrm{S}_{\mathrm{i}, \mathrm{t}}}{\mathrm{B}_{\mathrm{t}}}\right)+1\right), \\
\frac{\partial \mathrm{e}_{\mathrm{i}, \mathrm{t}}}{\partial \mathrm{B}_{\mathrm{t}}}=-\frac{\mathrm{S}_{\mathrm{i}, \mathrm{t}}}{\mathrm{B}_{\mathrm{t}} \cdot \mathrm{C}}, \\
\frac{\partial \mathrm{e}_{\mathrm{i}, \mathrm{t}}}{\partial \mathrm{C}}=-\frac{\mathrm{S}_{\mathrm{i}, \mathrm{t}}}{\mathrm{C}^{2}} \cdot \ln \left(\frac{S_{\mathrm{i}, \mathrm{t}}}{\mathrm{B}_{\mathrm{t}}}\right) .
\end{gathered}
$$

Eq. (4) then becomes:

$$
\begin{aligned}
\sigma\left(\mathrm{e}_{\mathrm{i}, \mathrm{t}}\right)=\left[\left(\frac{1}{\mathrm{C}} \cdot\left(\ln \left(\frac{\mathrm{S}_{\mathrm{i}, \mathrm{t}}}{\mathrm{B}_{\mathrm{t}}}\right)+1\right) \cdot \sigma\left(\mathrm{S}_{\mathrm{i}, \mathrm{t}}\right)\right)^{2}+\left(\frac{\mathrm{S}_{\mathrm{i}, \mathrm{t}}}{\mathrm{B}_{\mathrm{t}} \cdot \mathrm{C}}\right)^{2}\right. & \frac{\sum_{\mathrm{i}}\left(\mathrm{S}_{\mathrm{i}, \mathrm{t}} \cdot \sigma\left(\mathrm{S}_{\mathrm{i}, \mathrm{t}}\right)\right)^{2}}{\sum_{\mathrm{i}} \mathrm{S}_{\mathrm{i}, \mathrm{t}}^{2}} \\
& \left.+\left(\frac{\mathrm{s}_{\mathrm{i}, \mathrm{t}}}{\mathrm{C}^{2}} \cdot \ln \left(\frac{\mathrm{S}_{\mathrm{i}, \mathrm{t}}}{\mathrm{B}_{\mathrm{t}}}\right)\right)^{2} \cdot \sum_{\mathrm{t}}\left(\frac{\sum_{\mathrm{i}}\left(\mathrm{S}_{\mathrm{i}, \mathrm{t}} \cdot \sigma\left(\mathrm{S}_{\mathrm{i}, \mathrm{t}}\right)\right)^{2}}{\sum_{\mathrm{i}} \mathrm{S}_{\mathrm{i}, \mathrm{t}}{ }^{2}}\right)\right]^{\frac{1}{2}},
\end{aligned}
$$

and the full expression for the uncertainty in the image entropy is:

$$
\begin{aligned}
\sigma(\mathrm{E})=\left[\sum _ { \mathrm { t } } \sum _ { \mathrm { i } } \left(\left(\frac{1}{\mathrm{C}} \cdot\left(\ln \left(\frac{\mathrm{S}_{\mathrm{i}, \mathrm{t}}}{\mathrm{B}_{\mathrm{t}}}\right)+1\right) \cdot \sigma\left(\mathrm{S}_{\mathrm{i}, \mathrm{t}}\right)\right)^{2}\right.\right. & +\left(\frac{\mathrm{S}_{\mathrm{i}, \mathrm{t}}}{\mathrm{B}_{\mathrm{t}} \cdot \mathrm{C}}\right)^{2} \cdot \frac{\sum_{\mathrm{i}}\left(\mathrm{S}_{\mathrm{i}, \mathrm{t}} \cdot \sigma\left(\mathrm{S}_{\mathrm{i}, \mathrm{t}}\right)\right)^{2}}{\sum_{\mathrm{i}} \mathrm{S}_{\mathrm{i}, \mathrm{t}}{ }^{2}} \\
& \left.\left.+\left(\frac{\mathrm{S}_{\mathrm{i}, \mathrm{t}}}{\mathrm{C}^{2}} \cdot \ln \left(\frac{\mathrm{S}_{\mathrm{i}, \mathrm{t}}}{\mathrm{B}_{\mathrm{t}}}\right)\right)^{2} \cdot \sum_{\mathrm{t}}\left(\frac{\sum_{\mathrm{i}}\left(\mathrm{S}_{\mathrm{i}, \mathrm{t}} \cdot \sigma\left(\mathrm{S}_{\mathrm{i}, \mathrm{t}}\right)\right)^{2}}{\sum_{\mathrm{i}} \mathrm{S}_{\mathrm{i}, \mathrm{t}}{ }^{2}}\right)\right)\right]^{\frac{1}{2}} .
\end{aligned}
$$

To explain the dependency of Eq. (5) on relevant imaging parameters, several simplifying assumptions can be made. First, assuming the image noise is approximately constant over an ROI (i.e. $\sigma\left(\mathrm{S}_{\mathrm{i}, \mathrm{t}}\right) \approx \sigma(\mathrm{S})$ ) for all spatial and time indices, $\sigma(\mathrm{E})$ simplifies to give:

$$
\sigma(E) \approx \frac{\sigma(S)}{C} \sqrt{\sum_{t} \sum_{i}\left(\left(\ln \left(\frac{S_{i, t}}{B_{t}}\right)+1\right)^{2}+\left(\frac{S_{i, t}}{B_{t}}\right)^{2}+\left(\frac{S_{i, t} \cdot \ln \left(\frac{S_{i, t}}{B_{t}}\right)}{C}\right)^{2} \cdot T\right)},
$$

where $\mathrm{T}$ is the total number of time indices. Furthermore, the second and third terms of the above expression are experimentally small relative to the first, and can be removed: 


$$
\sigma(E) \approx \frac{\sigma(S)}{C} \sqrt{\sum_{t} \sum_{i}\left(\ln \left(\frac{S_{i, t}}{B_{t}}\right)+1\right)^{2}} .
$$

Finally, $\ln \left(\frac{S_{i, t}}{B_{t}}\right)<<0$ and so,

$$
\sigma(\mathrm{E}) \approx \frac{\sigma(\mathrm{S})}{\mathrm{C}} \sqrt{\sum_{\mathrm{t}} \sum_{\mathrm{i}}\left(\ln \left(\frac{\mathrm{S}_{\mathrm{i}, \mathrm{t}}}{\mathrm{B}_{\mathrm{t}}}\right)\right)^{2}} .
$$

The error in the image entropy is then inversely proportional to the signal-to-noise ratio, scaled by a second term. The scaling term is minimized when an image consists of a single bright pixel against a dark background. If the image is blurred, the scaling term grows, amplifying the uncertainty in the image entropy. Thus, the uncertainty in the image entropy is governed by the image noise but for images that are inherently blurry (poor resolution, misgating, etc) the contribution of the image noise to the uncertainty in the image entropy increases.

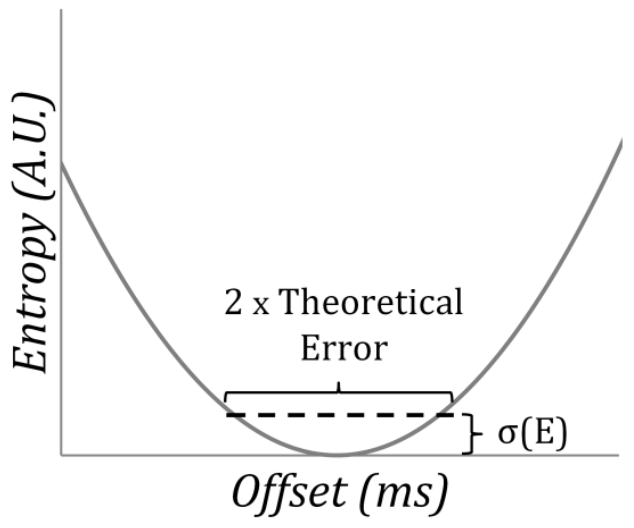

Figure 4 Entropy as a function of parameter offset. The theoretical error is given by the timing offset required to raise the entropy an amount $\sigma(\mathrm{E})$ above its minimum value.

The uncertainty in the image entropy is transformed into an uncertainty in each MOG parameter according to the following: first, MOG parameters are perturbed about their optimum values creating one-dimensional entropy landscapes (Figure 4). Second, the entropy landscapes are fit with a parabola. Finally, the uncertainty in each parameter is given by the change in parameter value required to raise the entropy an amount $\sigma(E)$ above its optimal value. The theoretical error is then dependent on the uncertainty in the 
image entropy and the curvature of the entropy landscape which, in turn, is dependent on the underlying motion and sensitivity of the image entropy to that motion.

\subsection{Methods}

\subsubsection{Adult Validation}

Validation of the MOG method for cine imaging of the heart was performed on healthy adult volunteers. Measurements were acquired with informed written consent and approval from the hospital research ethics board (REB). Short axis images of the heart were acquired on a 3.0T MRI research scanner (TRIO, Siemens Healthcare - Germany) using a multichannel cardiac coil. Three strategies were performed: Cartesian sampling without parallel imaging, Cartesian sampling with GRAPPA, and radial sampling without parallel imaging (38). A steady state free precession (SSFP) sequence was used for all image acquisitions (scan parameters listed in Table 2). The acquired spatial and temporal resolutions were chosen to provide coverage of the heart similar to that of the fetal acquisitions described below. For each scan, data were acquired under breath-hold and the ECG waveform was recorded for subsequent qualitative and quantitative comparison between ECG and MOG reconstructions.

MOG performance was evaluated by reconstructing the same data using either ECG trigger times or MOG parameters. The image quality of MOG reconstructions was compared against ECG reconstructions. Quantitative evaluation of MOG reconstructions was performed through an error analysis of the MOG parameters. An experimental error for MOG reconstructions was calculated from the standard-deviation of the difference between ECG trigger times and MOG parameters. Furthermore, the theoretical errors in the MOG parameters were calculated as described in the previous section.

\subsubsection{Fetal Evaluation}

Short axis images of the heart were acquired in a normal fetal subject (35 weeks gestational age) and another with a congenital heart defect (37 weeks gestational age). All fetal scans were performed on a 1.5T MRI clinical scanner (Avanto, Siemens Healthcare - 
Germany) using a multi-channel abdominal coil and a SSFP sequence (scan parameters listed in Table 2). The mother of the healthy fetal subject was recruited as part of a larger on-going study at our institution while the congenital heart defect patient was directed to MR to complement fetal echocardiography data that suggested fetal distress. In both cases, scans were performed with informed written consent and REB approval.

Table 2 Scan parameters for in vivo data acquisition.

\begin{tabular}{lccccc}
\hline & \multicolumn{3}{c}{ Adult } & \multicolumn{3}{c}{ Fetal } \\
\hline Field strength (T) & 3 & 3 & 3 & 1.5 & 1.5 \\
Trajectory & Cartesian & Cartesian & Radial & Cartesian & Cartesian \\
Parallel imaging & Off & GRAPPA & Off & Off & GRAPPA \\
Acceleration factor & N/A & 2 & N/A & N/A & 2 \\
Reference lines & N/A & 24 & N/A & N/A & 24 \\
Flip angle (degrees) & 45 & 45 & 45 & 56 & 56 \\
TE/TR (ms/ms) & $1.52 / 3.04$ & $1.52 / 3.04$ & $1.54 / 3.04$ & $1.33 / 3.14$ & $1.33 / 3.14$ \\
Views per segment & 15 & 15 & 15 & 8 & 16 \\
Temporal resolution (ms) & 46 & 46 & 46 & 25 & 50 \\
FOV (mm ) & $400 \times 396$ & $400 \times 396$ & $400 \times 400$ & $300 \times 220$ & $273 \times 340$ \\
Matrix size & $195 \times 192$ & $195 \times 192$ & $180 \times 192$ & $240 \times 176$ & $180 \times 224$ \\
Spatial resolution (mm ${ }^{2}$ ) & $2.1 \times 2.1$ & $2.1 \times 2.1$ & $2.1 \times 2.1$ & $1.3 \times 1.3$ & $1.5 \times 1.5$ \\
Number of slices & 1 & 1 & 1 & 1 & 10 \\
Slice thickness (mm) & 8 & 8 & 8 & 4 & 4 \\
Scan time per Slice (s) & 11 & 8 & 11 & 14 & 5 \\
\hline
\end{tabular}

Healthy fetal data were acquired under maternal breathhold in a single slice acquisition with no parallel imaging. These data were acquired to evaluate high spatial and temporal resolution fetal cardiac imaging with the MOG method. Accordingly, the scan length (14 s) does not necessarily reflect a practical clinical protocol where considerations given to fetal movement keep scan lengths as short as possible. For the images acquired in a fetal subject with a congenital heart defect, the scan length was shortened through the use of parallel imaging and the images were acquired with a free-breathing technique to allow adequate 
time for a multi-slice acquisition ( $5 \mathrm{~s}$ per slice). To minimize scan time and avoid fetal motion artifacts, the spatial and temporal resolution of the acquisition were reduced. As with the adult analysis described above, fetal data were evaluated both qualitatively and quantitatively. In the absence of an ECG gating signal for comparison, qualitative assessment of image quality was performed by an expert radiologist and quantitative evaluation of MOG performance was assessed by the theoretical error in the MOG parameters.

\subsubsection{MOG Algorithm}

All image reconstructions were performed offline on a personal computer using MATLAB (The Mathworks Inc - USA). The duration of each adult scan spanned 7-15 heartbeats and fetal scans spanned 15-30 heartbeats, resulting in hypothetical waveforms containing an equivalent range of parameters. The initial parameter values for the MOG search algorithm were based on a one dimensional (i.e., constant heart rate) brute force search to determine the mean heart rate. Optimization of individual R-wave triggers was then performed using the Nelder-Mead simplex algorithm, adapted for entropy minimization (67).

Entropy was calculated over user-defined ROIs, each prescribed using an initial temporallyaveraged image reconstructed from the oversampled data. Each ROI was chosen to cover the cardiac anatomy. For Cartesian sampling, the ROI extended approximately $20 \%$ beyond the heart in the phase encoding direction. This was done to capture ghosting artifact that would result from misgating, thereby improving the sensitivity of the MOG algorithm. Conversely, misgating artifact in radial sampling is isotropic, so ROIs were extended radially by approximately $20 \%$ beyond the heart for these data.

\subsection{Results}

\subsubsection{Adult Validation}

Figure 5 displays single slice short-axis images obtained in a healthy volunteer using three sampling strategies. For each sampling strategy, a comparison between ECG and MOG 
image reconstructions is shown for two cardiac phases (Figure 5a, c, e). Line plots of the left-ventricle signal intensity versus time (M-mode display) are also provided to compare the temporal dynamics of ECG and MOG reconstruction (Figure 5b, d, f). Qualitatively, these images show excellent agreement between ECG and MOG reconstructions across all sampling strategies. The agreement between ECG and MOG reconstructions was studied quantitatively by calculating the experimental error in the MOG trigger times relative to ECG. The experimental error was comparable for all three adult volunteer sampling strategies $( \pm 7 \mathrm{~ms})$ demonstrating that the MOG algorithm provided precise trigger times for reconstruction.

Figure 6 shows the theoretical error in each parameter for an adult Cartesian reconstruction and a radial MOG reconstruction. For brevity, only the theoretical errors for Cartesian and radial data without parallel imaging are shown; however, the theoretical errors for Cartesian data with parallel imaging exhibited a similar trend. Differences in the range of theoretical errors for Cartesian $( \pm 2-16 \mathrm{~ms})$ and radial $( \pm 8-11 \mathrm{~ms})$ data can be explained by the curvature of the entropy landscapes used to calculate the theoretical errors. Figure 7 shows representative one-dimensional entropy landscapes, relative to their optimal values, corresponding to the same data used to produce Figure 6. For the Cartesian data, landscape curvature varied across the acquisition, reflecting differences in the k-space data associated with each parameter. Because a linear-filling acquisition was used, central rows of k-space were collected near the midpoint of the scan and changes in the corresponding parameters resulted in greater curvature of the entropy landscape and consequently a smaller theoretical error. Conversely, landscape curvature was consistent between parameters for the radial acquisition and hence the theoretical errors shown in Figure 6 demonstrate less variation between parameter values when compared to Cartesian data. Despite the differences in relative curvature, all entropy landscapes were smooth with well-defined minima, supporting the use of the Nelder-Mead Simplex optimization method. 
Cartesian sampling without parallel imaging

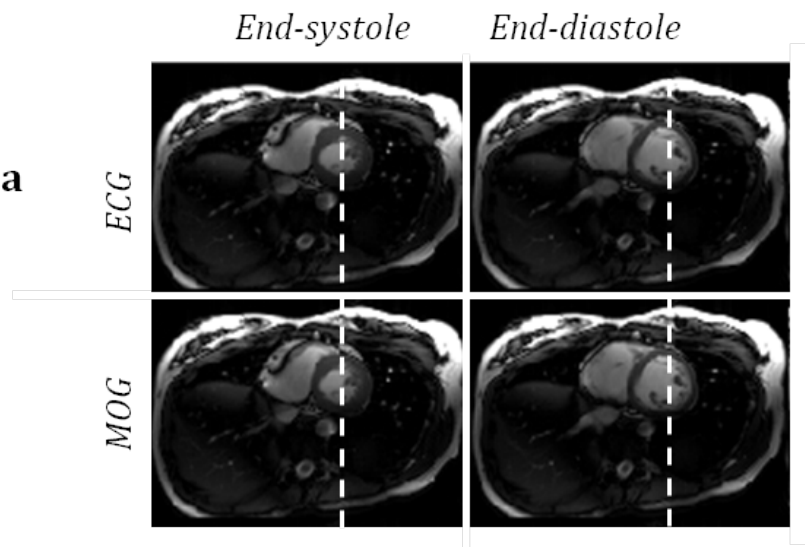

Cartesian sampling with GRAPPA

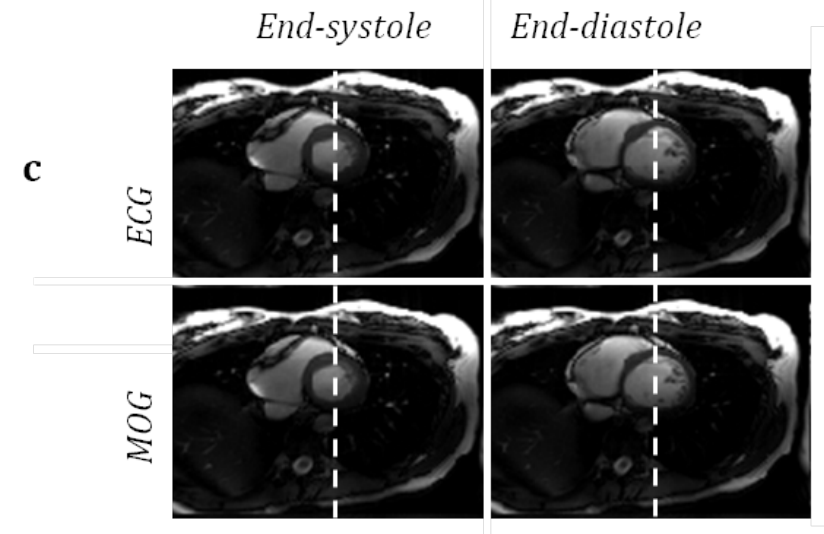

\section{Radial sampling without parallel imaging}

End-systole

End-diastole

e

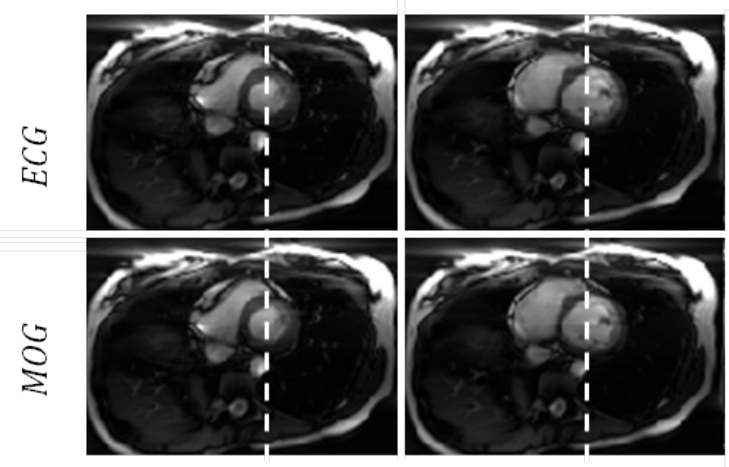

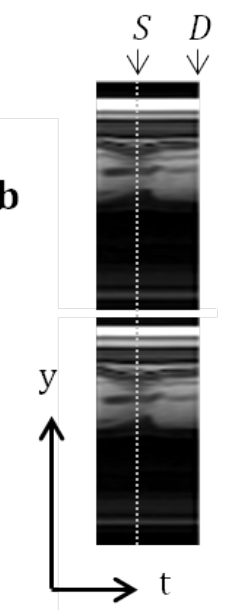

Figure 5: Comparison of MOG and ECG adult

volunteer

reconstructions. Endsystolic (S) and diastolic

(D) images are shown for each sampling strategy: (a, b) Cartesian without parallel imaging (c, d), Cartesian sampling with GRAPPA (e, f), and Radial sampling without parallel imaging. In all cases the temporal evolution of signal along the white dashed line is plotted. 
a Cartesian sampling

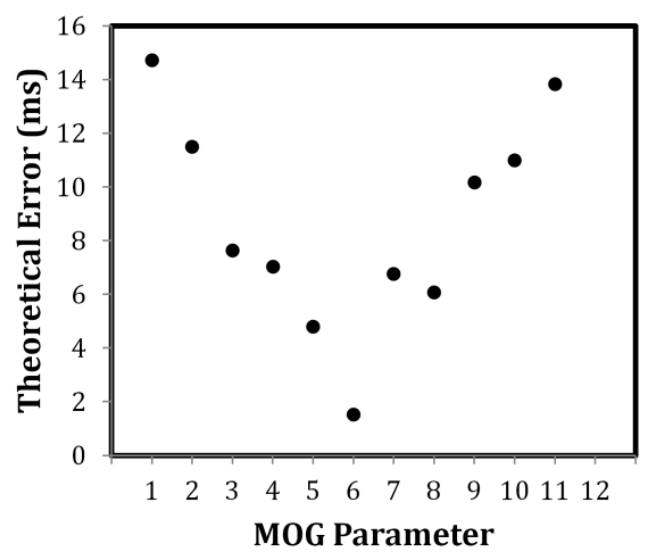

b Radial sampling

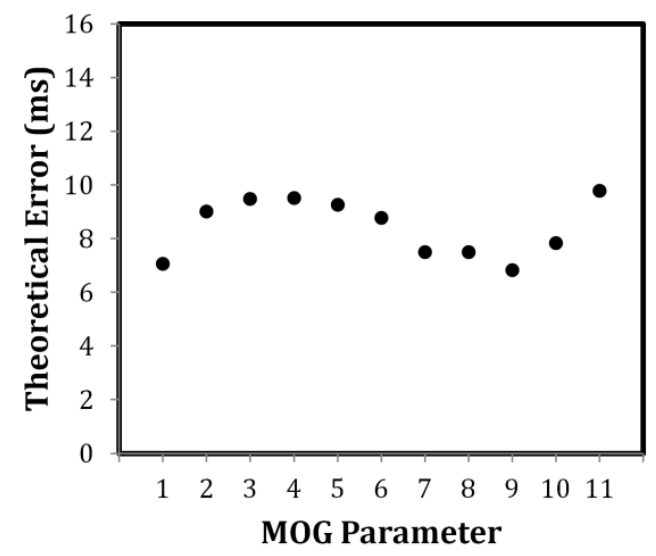

Figure 6: Theoretical error as a function of MOG parameter for data acquired using (a) Cartesian and (b) radial sampling.

Start of scan

(Parameter 1)
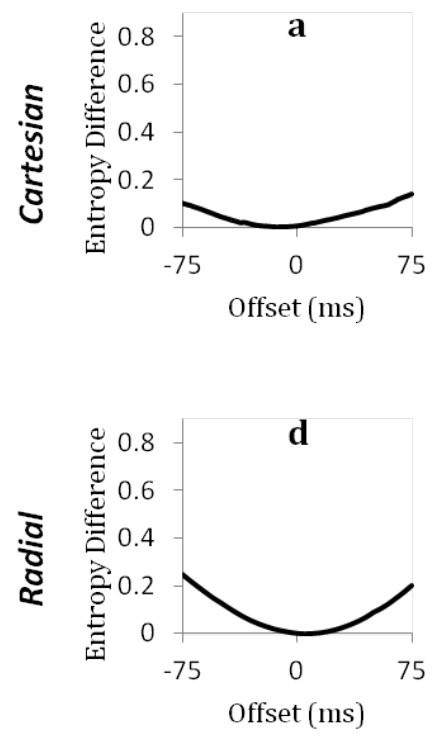

Midpoint of scan

(Parameter 6)
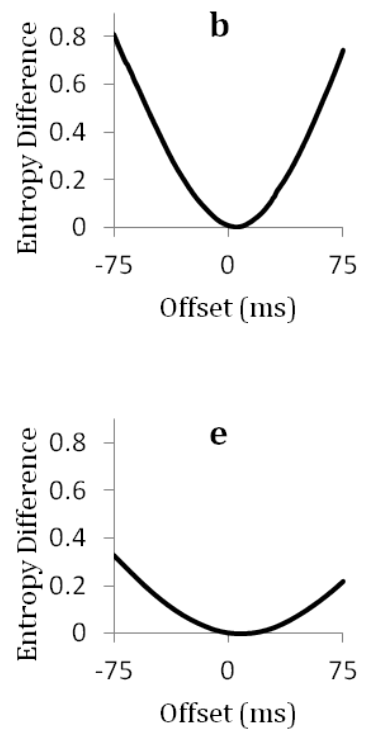

End of scan

(Parameter 11)
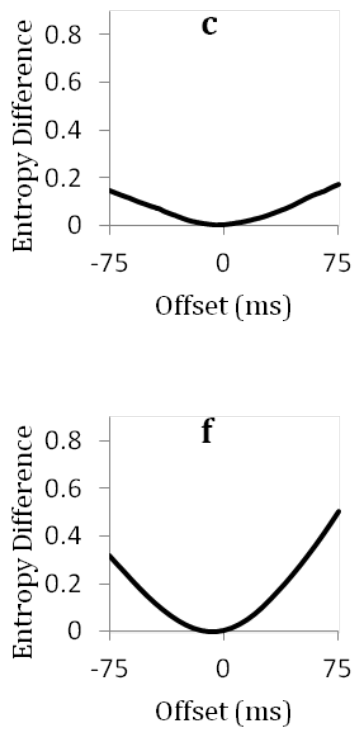

Figure 7: Entropy as a function of timing offset. (a-c) Demonstration of the change in entropy that occurs when Cartesian k-space data are interpolated and reconstructed based on R-wave triggers that are shifted from their true ECG values. (d-f) shows the same process but for radial data. 


\subsubsection{Fetal Evaluation}

Figure 8 displays short axis images of a healthy human fetal heart during end-systole and end-diastole. The cross-sections of the antero-lateral and postero-medial papillary muscles are visible in the bright blood pool of the left ventricle (arrow in Figure 8b). A normal thin rim of pericardial fluid appears as a thin bright band surrounding the two ventricles in the end-systolic images. The M-mode display shown in Figure 8c is comparable to the adult data displayed in Figure 5. Radial contraction and relaxation of the fetal ventricles is well represented and thus, ventricular function can be assessed from these images. Similarly, Figure 9 shows short axis images of the fetal heart for a subject with a congenital heart defect. Disproportionate left (arrow in Figure 9b) and right ventricles indicate a degree of left ventricular hypoplasia. Also, comparison of the end-systolic and end-diastolic images combined with the M-mode display in Figure 9c shows reduced contractility of the left ventricle when compared to that of the right ventricle. Aortic and mitral valve stenosis resulting in reduced filling and pressure overload of the left ventricle explain this ventricular dysfunction, which was confirmed postnatally in agreement with these MRI findings.

Although the lack of an external gating signal prevented direct measurement of experimental error in the MOG trigger times for fetal data, the theoretical errors in the optimized fetal parameters were calculated. The theoretical error in the fetal MOG parameters ranged from $\pm 3 \mathrm{~ms}$ to $\pm 34 \mathrm{~ms}$. As with the adult Cartesian data, the theoretical error in parameters that affected the central rows of K-space were lower than those affecting the periphery. 

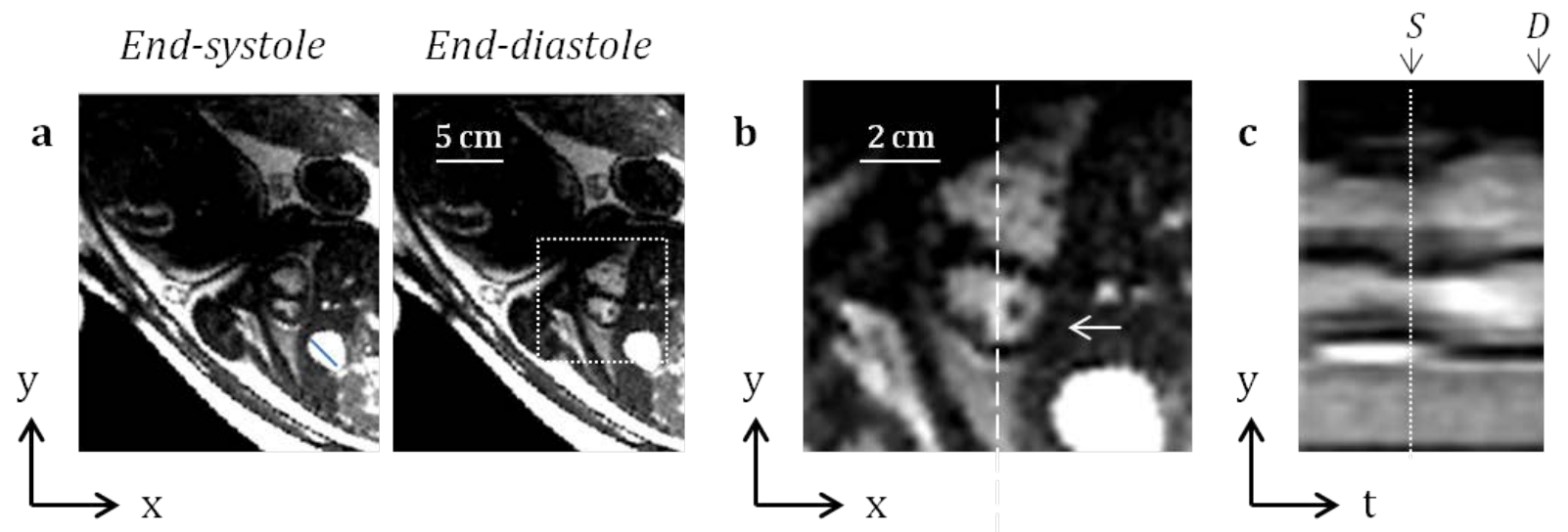

Figure 8: MOG reconstructions of a healthy human fetal heart. (a) A short axis view at the mid ventricular level is shown at end-systole and end-diastole. (b) Magnified end diastolic image from (a). (c) Plot of signal intensity versus time for the dashed line in (b).
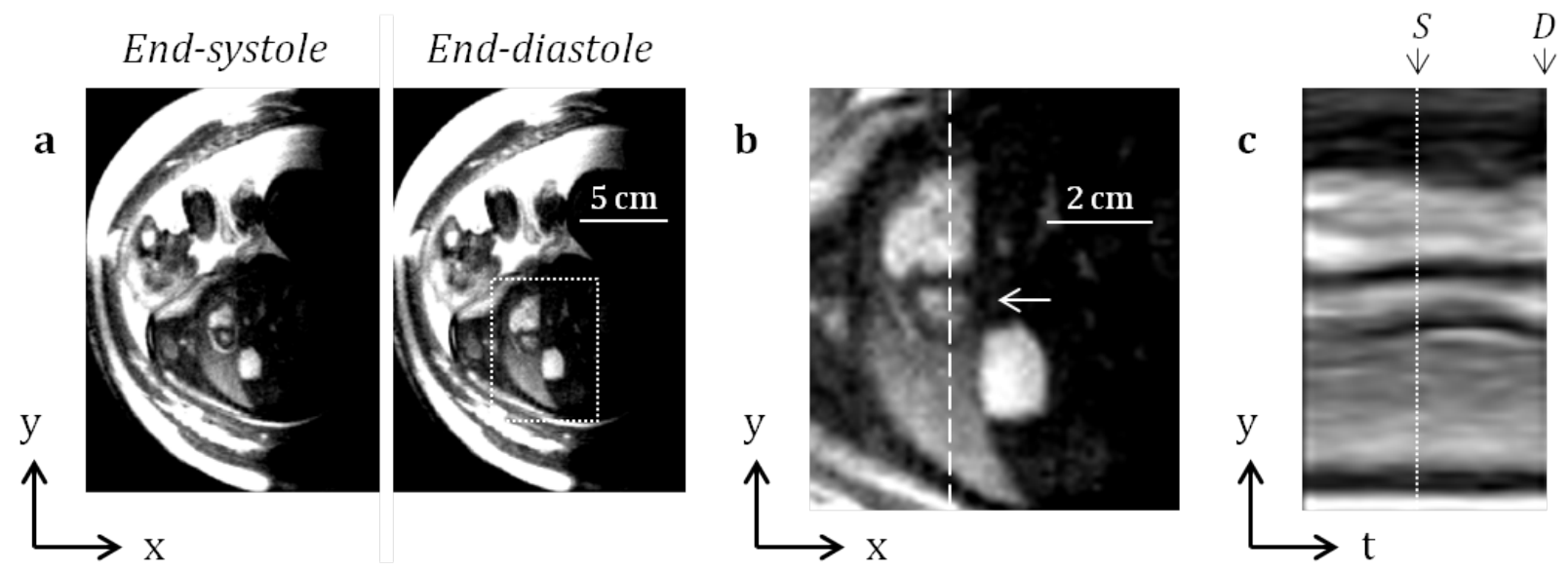

Figure 9: MOG reconstructions of human fetal subject with a congenital heart defect. (a) A short axis view at the mid ventricular level is shown at end-systole and end-diastole. (b) Magnified end diastolic image from (a). (c) Plot of signal intensity versus time for the dashed line in (b).

\subsection{Discussion}

In the previous section, metric optimized gating was developed and validated to retrospectively reconstruct oversampled MRI data for imaging fetal cardiac imaging. Using this technique, the first gated cine MR images of the human fetal heart were presented, 
demonstrating a new MR tool for assessing congenital heart disease and investigating cardiovascular compromise in other conditions such as placental insufficiency. Validation was performed in adults using short axis images of the heart acquired using three sampling strategies. Qualitative and quantitative analyses showed excellent agreement between MOG and ECG reconstructions of the same data.

\subsubsection{Validation}

The comparable quality of MOG and ECG image reconstructions in adults, combined with the precision of the optimized MOG trigger times (experimental error $\pm 7 \mathrm{~ms}$ ), provided a strong validation of the MOG technique for imaging the fetal heart. Furthermore, the theoretical error in adult MOG parameters ranged from $( \pm 2-16 \mathrm{~ms})$. The theoretical error was dependent on our ability to capture cardiac wall motion (temporal resolution), inherent image quality (SNR, spatial resolution), and metric sensitivity (see Theory section). The temporal resolution of the adult MR acquisition (46 ms) exceeded that needed for accurate interpolation of cardiac wall motion (i.e., the majority of cardiac motion is bounded by $\pm 20 \mathrm{~Hz}$ ) and thus, the theoretical error in the adult data was mainly a function of SNR and metric sensitivity (68-70).

\subsubsection{Fetal Application}

No study has been performed to determine the temporal bandwidth of human fetal cardiac motion and there is not yet a standard fetal CMR protocol. Therefore, a temporal resolution of $<40 \mathrm{~ms}$ was used to capture fetal end-systole (71). Still, it is possible that the current temporal resolution of the fetal scans may not fully capture fetal cardiac wall motion which, when combined with the lower SNR of the fetal scan, may explain the greater range of theoretical errors in fetal MOG parameters $( \pm 3-34 \mathrm{~ms}$ ) versus those for the adult Cartesian studies $( \pm 2-16 \mathrm{~ms})$. Nevertheless, the image quality of both fetal volunteer scans allowed for clinical assessment of cardiac function.

Finally, while radial acquisitions were performed in adults, fetal radial scans have yet to be performed. A comparison of Cartesian and radially acquired fetal data would be useful for 
determining the trade off between the potential benefits of non-Cartesian sampling (SNR, alternative acceleration strategies) and the potential increase in artifact from maternal anatomy.

\subsection{Conclusion}

In conclusion, a novel gating approach to CMR of the fetal heart was presented. Validation was performed in healthy adult volunteers both qualitatively and quantitatively. Qualitative evaluation was performed by comparing images reconstructed using MOG and ECG gating. Quantitative evaluation was performed through an experimental error analysis of the MOG triggers relative to those provided by ECG and a theoretical error analysis. With this method, the first gated high resolution dynamic images of the human fetal heart were acquired using conventional cine MRI techniques. 


\section{Chapter 3}

\section{Future Work}

\subsection{Introduction}

Until now, a method for gating MR images of the fetal heart was unavailable and consequently, cine images acquired in utero contained artifact due to misgating. In the previous chapter, MOG, a post-processing method for retrospectively gating cine MR images, was developed and validated for cine imaging of the heart. Using this technique, the first gated cine MR images of the human fetal heart were acquired. This development represents an important step towards MR evaluation of fetal cardiac function; however, fetal CMR is still subject to limitations. In this chapter, I outline the limitations of fetal CMR, and provide potential improvements to the MOG method. I suggest additional validation experiments for the presented application of MOG, discuss future application of fetal CMR using MOG, and conclude by discussing the future of fetal CMR.

\subsection{Limitations}

The fetal images presented in Chapter 2 suffer from a limitation of all fetal MRI studies: scan length. To avoid artifact from gross fetal movement, fetal MRI data are acquired as quickly as possible. This inherently constrains the available temporal and spatial resolution. Therefore, to improve fetal cardiac imaging, fetal cardiac gating (provided by MOG) must be combined with modern acceleration techniques to improve scan efficiency under an optimized fetal cardiac MRI protocol. Such a protocol will likely consist of a combination of parallel imaging and non-Cartesian sampling. I have shown through adult volunteer data that the MOG method is compatible with parallel imaging (GRAPPA) and non-Cartesian sampling (radial); however, these techniques involve a computationally intensive image reconstruction process which increases MOG search times. To address this, improvements to the MOG method are discussed in the next section. Specifically, improvements to the current entropy minimization process (Nelder-Mead simplex algorithm) may shorten post-processing time facilitating the use of modern acceleration techniques with MOG. 


\subsection{Improvements}

Despite the success of the MOG algorithm presented in Chapter 2, potential improvements have been identified. The following section outlines three aspects of the current implementation of the MOG method that may be improved: ROI selection, image metric, and search algorithm. Of these three aspects, improvements made to ROI selection and search algorithm may facilitate the implementation of MOG in a clinical setting while improvements made to metric selection may improve the error in MOG parameters.

The ROI used for the entropy calculation was user-defined, based on an initial temporally averaged reconstruction of the oversampled data. Potential segmentation solutions exist for removing this user-dependence, although the optimal ROI for MOG has not yet been investigated. The present approach to ROI selection attempts to maximize metric sensitivity by capturing dynamic fetal cardiac information within the ROI while avoiding possible artifacts from maternal cardiac or respiratory motion. Preliminary data (Figure 10 ) has shown that extending the ROI $\sim 20 \%$ beyond the cardiac anatomy increases the sensitivity of the image metric. This may be due to the sensitivity of the image entropy to ghosting that occurs outsie the cardiac anatomy. However, the orientation of the fetus in utero is unlikely to be consistent for each patient and therefore avoiding maternal artifact within the ROI may be particularly difficult for radial scans where artifacts are isotropic. Therefore, the current approach to ROI selection presents a guideline that may need to be optimized on a patient to patient basis.

Another potential improvement involves the image metric. In this work, the image entropy was used and demonstrated good sensitivity to artifact associated with misgated cine images of the heart. However, for both the Cartesian adult and fetal data presented in Chapter 2, the theoretical errors and hence metric sensitivity showed a dependence on the region of k-space affected by each parameter. For fetal data in particular, the SNR was much lower than that in adults and consequently the MOG parameters affecting the lowpowered periphery of k-space were less reliable than those affecting the relatively high 
powered central rows of k-space. Given a metric that is more sensitive to dynamic high resolution structures (i.e. entropy of the gradient) we may notice an improvement in the theoretical error of parameters affecting the outer rows of k-space (62). Having said that, the precision of the adult MOG parameters demonstrated in this work, combined with the excellent quality of fetal image reconstructions, suggests that improvements to the image acquisition (e.g., higher SNR and shorter scan duration) would provide greater benefit.

Finally, the MOG algorithm required full image reconstruction at each iteration so the entropy could be calculated over an ROI. In this work, the Nelder-Mead Simplex method was used and convergence was typically reached in 1000 iterations. For Cartesian sampled data without parallel imaging, MOG processing lasted 10 minutes per slice (MATLAB, standard desktop computer). For image reconstructions with a higher computational demand (parallel imaging, radial data), total MOG processing time lasted approximately 2 hours per slice. When compared to algorithms that calculate the gradient at some or all of the search points, this simplex approach decreased processing time, given that the calculation of a gradient would require several additional evaluations of the entropy function per iteration. Furthermore this method is orders of magnitude faster than a brute force approach. Still, while the Nelder-Mead Simplex method produced excellent results, further investigation into alternative optimization algorithms, computing hardware, and improved code efficiency may result in faster post-processing times, enabling the use of MOG in a clinical setting. Alternative optimization algorithms may involve a combination of the aforementioned methods to provide faster computational times for each iteration or faster convergence towards the global minimum.

\subsection{Further Investigation}

In this work, validation of the MOG method for cine imaging of the fetal heart focused on comparisons between MOG and ECG reconstructions of healthy adult volunteer data. While the theoretical error in MOG parameters provided insight into MOG performance for fetal reconstructions, a direct validation of fetal data was not performed. Future validation may be performed by extracting and comparing clinically relevant parameters from ultrasound 
and MOG reconstructions of fetal CMR data. However, while ultrasound is the standard imaging modality for overall in utero assessment of the fetal heart, it is not a gold standard for ventricular volumetry. Therefore, reproducibility studies of cardiac function as measured by fetal CMR data reconstructed using MOG may provide a better validation of the method. Regardless of the validation method chosen for future fetal studies, this method should be investigated over a broader population. In particular a MOG validation study performed in a neo-natal population would provide similar cardiac physiology to the late gestation fetus and allow for ECG gating.

a

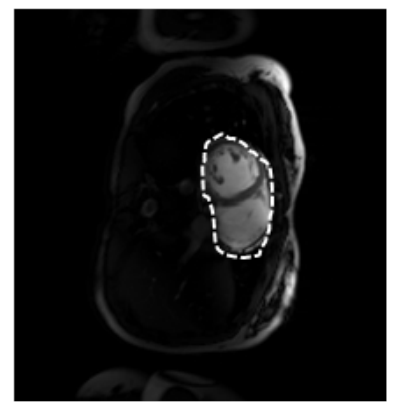

$\mathrm{b}$

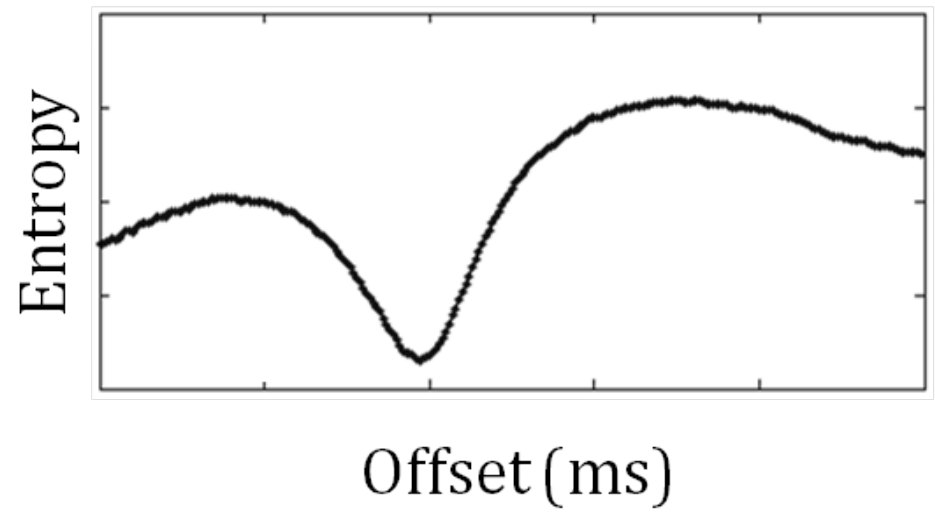

C

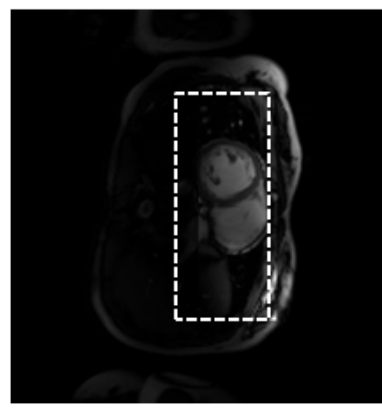

d

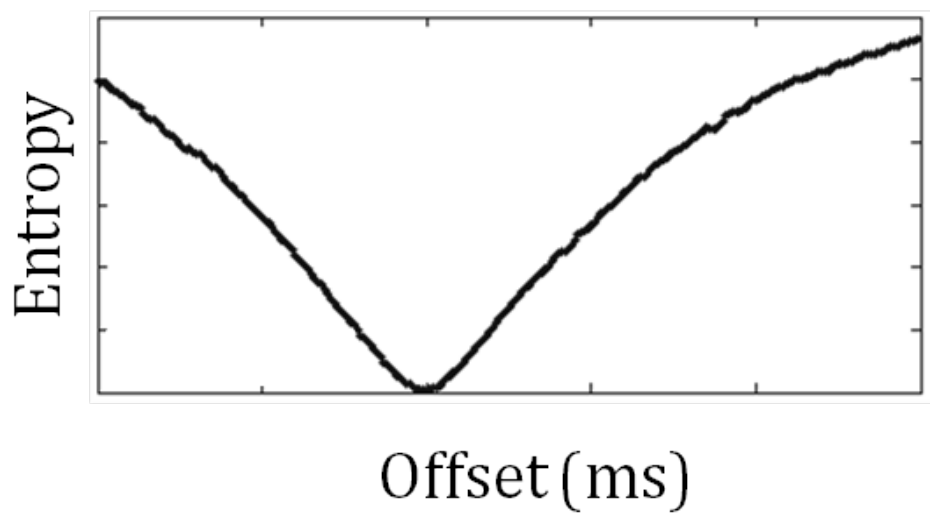

Figure 10: Preliminary results: ROI selection. (a) ROI example (dashed line), where the entropy is only evaluated over pixels that contain cardiac anatomy. (b) Demonstration of the change in entropy (evaluated over the ROI in a) that occurs when Cartesian k-space data are interpolated and reconstructed based on R-wave triggers that are shifted from their true ECG values. (c) ROI example (dashed line), where the entropy is evaluated over pixels extending $\sim 20 \%$ beyond the cardiac anatomy in either direction. (d) Same as (b) but for the ROI shown in (c). 


\subsection{Extensions}

Current applications of post-natal cardiac imaging with MRI include delineation of cardiac anatomy and evaluation of cardiac function through ventricular volumetry (72-75). The use of MOG will facilitate the translation of these and other applications to the fetal population. For example, extending MOG to other cine imaging sequences should be relatively straight forward. In particular, the use of a gradient echo sequence may allow for the application of DESPOT1, a $\mathrm{T}_{1}$ mapping technique, to fetal imaging (76).

Furthermore, MOG may complement existing gating strategies in both fetal and adult populations. For example, if an external gating signal is unreliable because it is weak (fetal ECG, self-gating, high field MRI) or erratic (cardiac arrhythmia), oversampled data could be collected together with the external gating signal, and the data could be retrospectively sorted using MOG to modify the unreliable triggers.

\subsection{The Future of Fetal Imaging}

Considerations given to cost and availability will likely prevent fetal CMR from becoming a regular screening tool. However, in high risk pregnancies with suspected fetal distress, choices regarding delivery and antenatal or perinatal treatment require precise quantitative assessment of the cardiac anatomy, blood flow, and oxygen delivery. Therefore CMR may become an important tool, providing information that cannot be obtained with echocardiography alone. Furthermore, during late gestation, when decisions regarding the timing of the delivery become important, the feasibility of CMR increases while that of echocardiography decreases due to the size of the fetus. A large fetus may limit ultrasonic assessment due to occluded cardiac anatomy from acoustic shadowing. Conversely, a large fetus is often less mobile and thus considerations given to scan length may be reduced. Lastly, when compared to echocardiography, the accuracy provided by an MRI assessment of fetal cardiac anatomy and blood flow may lend itself to research applications including both clinical studies of antenatal treatments and new insights into the normal fetal circulation. 


\subsection{Final Remarks}

In conclusion, I have demonstrated that MOG can be used to reliably reconstruct cine images of the fetal heart without a gating signal. There is increasing interest in the use of MRI for studying fetal hemodynamics and as such the technique presented in this work, combined with previous work in MOG for PC-MRI measurements of fetal blood flow, builds towards a comprehensive in utero assessment of the fetal circulation. Still, there remain significant challenges in adapting these applications to the clinical environment. Constraints on scan length, due to the possibility of fetal motion combined with the size of fetal cardiac structures and the speeds at which they move, necessitate new approaches, as the temporal and spatial resolutions provided by current fetal imaging protocols restrict high quality cardiac imaging to late gestation or the use of sedation to prevent gross fetal motion. Nevertheless, the development of a gating technique for fetal CMR is an important step towards high quality imaging that can not only capture the dynamics of the fetal cardiac cycle, but provide new methods for studying diseases that disrupt the fetal circulation. 


\section{References}

1. Kim D-H, Chung S, Vigneron DB, Barkovich AJ, Glenn OA. Diffusion-weighted imaging of the fetal brain in vivo. Magn Reson Med. 2008 Jan;59(1):216-20.

2. Kim D-hyun, Vahidi K, Caughey AB, Coakley FV, Vigneron DB, Kurhanewicz J, et al. In vivo (1)H magnetic resonance spectroscopy of amniotic fluid and fetal lung at $1.5 \mathrm{~T}$ : technical challenges. J Magn Reson Imaging. 2008 Oct;28(4):1033-8.

3. Sørensen A, Holm D, Pedersen M, Tietze A, Stausbøl-Grøn B, Duus L, et al. Left-right difference in fetal liver oxygenation during hypoxia estimated by BOLD MRI in a fetal sheep model. Ultrasound Obstet Gynecol. 2011 Dec;38(6):665-72.

4. Cannie M, Jani J, Dymarkowski S, Deprest J. Fetal magnetic resonance imaging: luxury or necessity? Ultrasound Obstet Gynecol. 2006 May;27(5):471-6.

5. Parer JT, Livingston EG. What is fetal distress? Am J Obstet Gynecol. 1990;162(6):1421-7.

6. Loftin RW, Habli M, Snyder CC, Cormier CM, Lewis DF, Defranco EA. Late preterm birth. Review of Obstetrics and Gynecology. 2010;3(1):10-9.

7. Information CI for H. Hightlights of 2009-2010 Selected Indicators Describing the Birthing Process in Canada. 2011.

8. Practise $\mathrm{C}$ on O. Fetal distress and birth asphyxia. ACOG Committee Opinion. Int J Gynaecol Obstet. 1994;45(3):302.

9. Behrman RE, Butler AS, (U.S.) I of M. Committee on Understanding Premature Birth and Assuring Healthy Outcomes. Washington, D.C.: National Academies Press; 2007.

10. Kramer MS, Demissie K, Yang H, Platt RW, Sauve R, Liston R. The contribution of mild and moderate preterm birth to infant mortality. Fetal and INfant Health Study Group of the Canadian Perinatal Surveillance System. JAMA. 2000;284(7):8430849. 
11. Rudolph AM. Congenital Diseases of the Heart: Clinical-Physiological Considerations. 3rd ed. Wiley Blackwell; 2009.

12. Kiserud T, Rasmussen S, Skulstad S. Blood flow and the degree of shunting through the ductus venosus in the human fetus. Am J Obstet Gynecol. 2000 Jan;182(1 Pt 1):147-53.

13. Uflacker R. Atlas of Vascular Anatomy: An Angiographic Approach. 2nd ed. Am Med Assoc; 2007.

14. Godfrey ME, Messing B, Cohen SM, Valsky DV, Yagel S. Functional assessment of the fetal heart: a review. Ultrasound Obstet Gynecol. 2012 Feb;39(2):131-44.

15. Allan LD, Joseph MC, Boyd EG, Campbell S, Tynan M. M-mode echocardiography in the developing human fetus. Br Heart J. 1982 Jun;47(6):573-83.

16. Allan LD, Chita SK, Al-Ghazali W, Crawford DC, Tynan M. Doppler echocardiographic evaluation of the normal human fetal heart. Heart. 1987 Jun 1;57(6):528-33.

17. Uittenbogaard LB, Haak MC, Spreeuwenberg MD, van Vugt JMG. Fetal cardiac function assessed with four-dimensional ultrasound imaging using spatiotemporal image correlation. Ultrasound Obstet Gynecol. 2009 Mar;33(3):272-81.

18. Quinones MA, Pickering E, Alexander JK. Percentage of shortening of the echocardiographic left ventricular dimension. Its use in determining ejection fraction and stroke volume. Chest. 1978 Jul;74(1):59-65.

19. Colocousis JS, Huntsman LL, Curreri PW. Estimation of stroke volume changes by ultrasonic doppler. Circulation. 1977 Dec;56(6):914-7.

20. Simioni C, Marcondes L, Nardozza M, Rolo LC, Zamith M, Ju EA, et al. Heart stroke volume , cardiac output, and ejection fraction in 265 normal fetus in the second half of gestation assessed by 4D ultrasound using spatio-temporal image correlation. Heart. 2011;(August 2010):1-9. 
21. Mielke G, Benda N. Cardiac output and central distribution of blood flow in the human fetus. Circulation. 2001 Mar 27;103(12):1662-8.

22. Gill RW. Measurement of Blood Flow by Ultrasound: Accuracy and Sources of Error. Ultrasound in Medicine and Biology. 1985;I(4):625-41.

23. Sklansky M. Advances in Fetal Cardiac Imaging. Pediatric Cardiology. 2004;:307-21.

24. Manganaro L, Savelli S, Maurizio MD, Perrone A, Francioso A, Barbera LL, et al. Assessment of congenital heart disease ( CHD ): Is there a role for fetal magnetic resonance imaging ( MRI )? European Journal of Radiology. 2009;72:172-80.

25. Mieghem TV, Dekoninck P, Steenhaut P, Deprest J. Methods for prenatal assessment of fetal cardiac function. Review Literature And Arts of The Americas. 2009;(June):1193-203.

26. Smith FW, Adam AH, Phillips WD. NMR imaging in pregnancy. Lancet. 1983 Jan 1;1(8314-5):61-2.

27. Baker P, Johnson I, Gowland P, Hykin J, Harvey P, Freeman A, et al. Fetal weight estimation by echo-planar magnetic resonance imaging. The Lancet. 1994;343(8898):644-5.

28. Baker PN, Johnson IR, Gowland PA, Freeman A, Adams V, Mansfield P, et al. Estimation of fetal lung volume using echo-planar magnetic resonance imaging. Obstet Gynecol. 1994;83(6):951.

29. Baker PN, Johnson IR, Gowland P a, Hykin J, Adams V, Mansfield P, et al. Measurement of fetal liver, brain and placental volumes with echo-planar magnetic resonance imaging. Br J Obstet Gynaecol. 1995 Jan;102(1):35-9.

30. Lotz J, Meier C, Leppert A, Galanski M. Cardiovascular flow measurement with phasecontrast MR imaging: basic facts and implementation. Radiographics. 2002;22(3):651-71. 
31. Møgelvang J, Stubgaard M, Thomsen C, Henriksen O. Evaluation of right ventricular volumes measured by magnetic resonance imaging. Eur Heart J. 1988 May;9(5):52933.

32. Nield LE, Qi X-LL, Valsangiacomo ER, Macgowan CK, Wright GA, Hornberger LK, et al. In vivo MRI measurement of blood oxygen saturation in children with congenital heart disease. Pediatr Radiol. 2005 Feb;35(2):179-85.

33. Wedega U, Kooijman H, Yamamura J, Frisch M, Weber C, Buchert R, et al. In Vivo MRI Measurement of Fetal Blood Oxygen Saturation in Cardiac Ventricles of Fetal Sheep : A Feasibility Study. 2010;41:32-41.

34. DeVore GR. Assessing fetal cardiac ventricular function. Semin Fetal Neonatal Med. 2005 Dec;10(6):515-41.

35. Sandmire HF, DeMott RK. Electronic fetal heart rate monitoring: research guidelines for interpretation. Am J Obstet Gynecol. 1998 Jul;179(1):276-7.

36. Markl M. Phase contrast MRI with improved temporal resolution by view sharing: $\mathrm{k}-$ space related velocity mapping properties. Magnetic Resonance Imaging. $2001 ; 19: 669-676$.

37. Glockner JF, Hu HH, Stanley DW, Angelos L, King K. Parallel MR Imaging: A User's Guide. Radiographics. 2005;25(5):1279-97.

38. Griswold MA, Jakob PM, Heidemann RM, Nittka M, Jellus V, Wang J, et al. Generalized autocalibrating partially parallel acquisitions (GRAPPA). Magn Reson Med. 2002 Jun;47(6):1202-10.

39. Pruessmann KP, Weiger M, Scheidegger MB, Boesiger P. SENSE : Sensitivity Encoding for Fast MRI. Knowledge Creation Diffusion Utilization. 1999;962:952-62.

40. Lustig M, Donoho D, Pauly JM. Sparse MRI : The Application of Compressed Sensing for Rapid MR Imaging. Magnetic Resonance in Medicine. 2007;1195:1182-95. 
41. Zhao L, Madore B, Panych LP. Reduced field-of-view MRI with two-dimensional spatially-selective RF excitation and UNFOLD. Magn Reson Med. 2005 May;53(5):1118-25.

42. Kim D, Dyvorne HA, Otazo R, Feng L, Sodickson DK, Lee VS. Accelerated PhaseContrast Cine MRI Using k-t. Magnetic Resonance in Medicine. 2011;000:1-11.

43. Stalder F, Bauer S, Markl M, Jung B. On the Undersampling Strategies to Accelerate Time-Resolved 3D Imaging Using k-t-GRAPPA. Magnetic Resonance in Medicine. 2011;975:966-75.

44. Liang D, Liu B, Wang J, Ying L. Accelerating SENSE Using Compressed Sensing. Magnetic Resonance in Medicine. 2009;1584:1574 -1584.

45. Lanzer P, Botvinick E, Schiller N, Crooks L, Arakawa M, Kaufman L, et al. Cardiac imaging using gated magnetic resonance. Radiology. 1984;150(1):121.

46. Lenz GW, Haacke EM, White RD. Retrospective cardiac gating: A review of technical aspects and future directions. Magnetic Resonance Imaging. 1989 Sep;7(5):445-55.

47. Brugger PC. Fetal MRI. Berlin, Heidelberg: Springer Berlin Heidelberg; 2011.

48. Peters M, Crowe J, Piéri JF, Quartero H, Hayes-Gill B, James D, et al. Monitoring the fetal heart non-invasively: a review of methods. J Perinat Med. 2001 Jan;29(5):40816.

49. Feinberg D a, Giese D, Bongers DA, Ramanna S, Zaitsev M, Markl M, et al. Hybrid ultrasound MRI for improved cardiac imaging and real-time respiration control. Magn Reson Med. 2010 Feb;63(2):290-6.

50. Yamamura J, Kopp I, Frisch M, Fischer R, Valett K, Hecher K, et al. Cardiac MRI of the fetal heart using a novel triggering method: initial results in an animal model. J Magn Reson Imaging. 2012 May;35(5):1071-6. 
51. Spraggins TA. Wireless retrospective gating: application to cine cardiac imaging. Magn Reson Imaging. 1990 Jan;8(6):675-81.

52. Crowe ME, Larson AC, Zhang Q, Carr J, White RD, Li D, et al. Automated rectilinear self-gated cardiac cine imaging. Magn Reson Med. 2004 Oct;52(4):782-8.

53. Thompson RB, McVeigh ER. Flow-gated phase-contrast MRI using radial acquisitions. Magn Reson Med. 2004 Sep;52(3):598-604.

54. Larson AC, White RD, Laub G, McVeigh ER, Li D, Simonetti OP. Self-gated cardiac cine MRI. Magn Reson Med. 2004 Jan;51(1):93-102.

55. Hiba B, Richard N, Thibault H, Janier M. Cardiac and respiratory self-gated cine MRI in the mouse: comparison between radial and rectilinear techniques at 7T. Magn Reson Med. 2007 Oct;58(4):745-53.

56. Holmes WM, McCabe C, Mullin JM, Condon B, Bain MM. Images in cardiovascular medicine. Noninvasive self-gated magnetic resonance cardiac imaging of developing chick embryos in ovo. Circulation. 2008 May 27;117(21):e346-7.

57. Yamamura J, Frisch M, Ecker H, Graessner J, Hecher K, Adam G, et al. Self-gating MR imaging of the fetal heart: comparison with real cardiac triggering. Eur Radiol. 2011 Jan;21(1):142-9.

58. Seiberlich N, Lee G, Ehses P, Duerk JL, Gilkeson R, Griswold M. Improved Temporal Resolution in Cardiac Imaging Using Through-Time Spiral GRAPPA. In Vivo. 2011;1688(April):1682-8.

59. Haacke EM, Lenz GW, Nelson AD. Pseudo-gating: elimination of periodic motion artifacts in magnetic resonance imaging without gating. Magn Reson Med. 1987 Feb;4(2):162-74. 
60. Jansz MS, Seed M, van Amerom JFP, Wong D, Grosse-Wortmann L, Yoo S-J, et al. Metric optimized gating for fetal cardiac MRI. Magn Reson Med. 2010 Nov;64(5):1304-14.

61. Atkinson D, Hill DL, Stoyle PN, Summers PE, Keevil SF. Automatic correction of motion artifacts in magnetic resonance images using an entropy focus criterion. IEEE Trans Med Imaging. 1997 Dec;16(6):903-10.

62. McGee KP, Manduca A, Felmlee JP, Riederer SJ, Ehman RL. Image metric-based correction (autocorrection) of motion effects: analysis of image metrics. J Magn Reson Imaging. 2000 Feb;11(2):174-81.

63. Manduca A, McGee K, Welch E, Felmlee J, Ehman RL. Autofocusing of clinical shoulder MR images for correction of motion artifacts. Medical Image Computing and Computer-Assisted Interventation — MICCAI'98. 1998;:598-605.

64. McGee KP, Felmlee JP, Jack CR, Manduca A, Riederer SJ, Ehman RL. Autocorrection of three-dimensional time-of-flight MR angiography of the Circle of Willis. AJR Am J Roentgenol. 2001 Feb;176(2):513-8.

65. McGee K, Felmlee J, Ehman R. Autocorrection of MR images using multiple metrics. US Patent 6,265,874. 2001;

66. Bevington P, Robinson DK. Data Reduction and Error Analysis for the Physical Sciences. 2nd ed. McGraw-Hill; 1994.

67. Lagarias J, Reeds J, Wright M. Convergence properties of the Nelder-Mead simplex method in low dimensions. Siam journal of. 1998;9(1):112-47.

68. Foo TK, Bernstein MA, Aisen AM, Hernandez RJ, Collick BD, Bernstein T. Improved ejection fraction and flow velocity estimates with use of view sharing and uniform repetition time excitation with fast cardiac techniques. Radiology. 1995 May;195(2):471-8. 
69. Holdsworth DW, Norley CJ, Frayne R, Steinman DA, Rutt BK. Characterization of common carotid artery blood-flow waveforms in normal human subjects. Physiol Meas. 1999 Aug;20(3):219-40.

70. Kramer CM, Barkhausen J, Flamm SD, Kim RJ, Nagel E. Standardized cardiovascular magnetic resonance imaging (CMR) protocols, society for cardiovascular magnetic resonance: board of trustees task force on standardized protocols. J Cardiovasc Magn Reson. 2008 Jan;10:35.

71. Tsyvian P, Malkin K, Wladimiroff JW. Assessment of fetal left cardiac isovolumic relaxation time in appropriate and small-for-gestational-age fetuses. Ultrasound Med Biol. 1995 Jan;21(6):739-43.

72. Thiele H, Nagel E, Paetsch I, Schnackenburg B, Bornstedt A, Kouwenhoven M, et al. Functional cardiac MR imaging with steady-state free precession (SSFP) significantly improves endocardial border delineation without contrast agents. J Magn Reson Imaging. 2001 0ct;14(4):362-7.

73. Rathi VK, Biederman RWW. Imaging of ventricular function by cardiovascular magnetic resonance. Curr Cardiol Rep. 2004 Jan;6(1):55-61.

74. Alfakih K, Reid S, Jones T, Sivananthan M. Assessment of ventricular function and mass by cardiac magnetic resonance imaging. Eur Radiol. 2004 Oct;14(10):1813-22.

75. Peshock RM, Willett DL, Sayad DE, Hundley WG, Chwialkowski MC, Clarke GD, et al. Quantitative MR imaging of the heart. Magn Reson Imaging Clin N Am. 1996 May;4(2):287-305.

76. Deoni SCL, Peters TM, Rutt BK. High-resolution T1 and T2 mapping of the brain in a clinically acceptable time with DESPOT1 and DESPOT2. Magn Reson Med. 2005 Jan;53(1):237-41. 


\section{Copyright Acknowledgements}

Figure 1 was adapted from "Atlas of Vascular Anatomy: An Angiographic Approach", written by Renan Uflacker and published by Lippincott Williams and Wilkins. 